\title{
Avoiding observability singularities in output feedback bilinear systems
}

\author{
Lucas Brivadis $^{1}$, Jean-Paul Gauthier ${ }^{2}$, Ludovic Sacchelli ${ }^{1}$, and Ulysse Serres ${ }^{1}$ \\ ${ }^{1}$ Univ. Lyon, Université Claude Bernard Lyon 1, CNRS, LAGEPP UMR 5007, 43 bd \\ du 11 novembre 1918, F-69100 Villeurbanne, France \\ ${ }^{2}$ Université de Toulon, Aix Marseille Univ, CNRS, LIS, France
}

Tuesday $12^{\text {th }}$ January, 2021

\begin{abstract}
Control-affine output systems generically present observability singularities, i.e. inputs that make the system unobservable. This proves to be a difficulty in the context of output feedback stabilization, where this issue is usually discarded by uniform observability assumptions for state feedback stabilizable systems. Focusing on state feedback stabilizable bilinear control systems with linear output, we use a transversality approach to provide perturbations of the stabilizing state feedback law, in order to make our system observable in any time even in the presence of singular inputs.
\end{abstract}

Keywords: Observability, Transversality theory, Output feedback, Stabilization

\section{Introduction}

Stabilizing the state of a dynamical system to a target point is a classical problem in control theory. However, in many physical problems, only part of the state is known. Hence a state feedback can not be directly implemented. When a stabilizing state feedback exists, a commonly used idea is to apply this feedback to an estimation of the state, relying on a dynamical system called the observer, which learns the state of the system from its dynamics and the measured output. This strategy belongs to the family of dynamic output feedback stabilization techniques.

In the deterministic setting, output feedback stabilization has been extensively studied (see e.g. 2, 3, 6, 7, 9, 13, 15, 17, 18,). The observability of a controlled system for some fixed input qualifies the ability to estimate the state using its output, and characterizes the fact that two trajectories of the system can be distinguished by their respective outputs over a given time interval. This crucial notion constitutes a field of study in itself (see e.g. [2, 4, 10, 19]). A commonly used hypothesis to achieve output feedback stabilization is the uniform observability of the system, that is the system is observable for all possible inputs. It is well-known that a globally state feedback stabilizable system that is uniformly observable is also semi-globally output feedback stabilizable (see e.g. [7, 13, 17, 18]).

However, as shown in [10], it is not generic for a dynamical system to be uniformly observable. There may exist singular inputs for the system, that are inputs that make the system unobservable on any time interval, and the output feedback may produce such singular inputs. This defeats the purpose of output feedback stabilization, which is still an open problem when 
such inputs exist. Investigating this issue, some authors propose a different approach by allowing time-varying (either periodic as in [6] or "sample and hold" as in [16]) output feedback. Doing so, the authors use a separation principle to show output feedback stabilization. Adopting another point of view and in line with [14, we are interested in smooth time-invariant output feedback.

In this work, we restrict ourselves to the class of single-input single-output bilinear systems with linear observation that are state feedback stabilizable at some target point, which, with no loss of generality, is chosen to be 0 . We also assume the system to be observable at the target, that is, the constant input obtained by evaluation of the feedback at 0 is not singular. This class of systems is a natural choice of study for two reasons. First, the uniform observability hypothesis is still not generic in this case. In particular, one can easily check that there generically exists constant inputs that make the system unobservable in any time. Secondly, according to [8], any control-affine system with finite dimensional observation space may be immersed in such a system.

In this context, a natural question to ask is: "Can we ensure that only observable inputs are produced by the dynamics when the output feedback is obtained as a combination of an observer and a stabilizing state feedback?" This question falls within the more general and unsolved problem of building a smooth separation principle for systems with observability singularities. One cannot hope for generic bilinear systems that all stabilizing state feedback laws ensure the observability of the closed-loop system. However, we show that for any stabilizing state feedback law, there exist small additive perturbations to this feedback that satisfy this observability property and conserve its locally stabilizing property. Transversality theory is used to prove the existence of such an open and dense class of perturbations. In particular, for almost all considered systems, almost any locally stabilizing feedback law ensures observability of the closed-loop system. Stabilization by output feedback is beyond the scope of this paper, which focuses only on the observability issue. Yet, the obtained results may pave the way to the construction of a "generic" separation principle. For our results to hold, some properties of the dynamical observer are needed. The problem is tackled with a general observer design, and it is shown in a closing section that the classical Luenberger and Kalman observers fit our hypotheses.

\section{Organization of the paper}

In Section 2, we state the main results of this paper. We begin this section with some definitions and notations, and we emphasise the precise issue. In particular, we define the system and the class of feedback perturbations we are interested in. We then state our main results on observability properties of the perturbed system, and assert that the classical Kalman and Luenberger observers fit our hypotheses.

In Section 3 the reader may find a proof of our main results in three subsections. We rely on a transversality approach, which requires some technical preliminary results (Section 3.1). Sections 3.2 and 3.3 are then focused on the proof of our first main theorem and its corollary, respectively.

Lastly, we prove in Section 4 that the Luenberger and Kalman observers fit our hypotheses, so that we can apply our previous theorems to these observers. In order to do so, we prove that their dynamics are somehow compatible with the Kalman observability decomposition.

\section{Notations}

Let $\mathbb{N}$ be the set of non-negative integers. For any subset $\mathcal{I} \subset \mathbb{N},|\mathcal{I}|$ denotes its cardinality. 
Let $n, m$ be positive integers. Let $\langle\cdot, \cdot\rangle$ be the canonical scalar product on $\mathbb{R}^{n},|\cdot|$ the induced Euclidean norm, $B(x, r)$ the open ball centered at $x$ of radius $r$ for this norm, and $\mathbb{S}^{n-1} \subset \mathbb{R}^{n}$ the unit sphere. Let $\mathcal{L}\left(\mathbb{R}^{n}, \mathbb{R}^{m}\right)$ be the set of linear maps from $\mathbb{R}^{n}$ to $\mathbb{R}^{m}$ and $\operatorname{End}\left(\mathbb{R}^{n}\right)=\mathcal{L}\left(\mathbb{R}^{n}, \mathbb{R}^{n}\right)$. For any endomorphism $A \in \operatorname{End}\left(\mathbb{R}^{n}\right)$, denote by $A^{*}$ its adjoint operator.

If $f$ is a function from $\mathbb{R}^{n}$ to $\mathbb{R}^{m}$, the notation $D f(x)[v]$ stands for the differential at $x \in \mathbb{R}^{n}$ applied to the vector $v \in \mathbb{R}^{n}$ of the function $f$. The partial differential of $f$ at $x$ with respect to the variable $y$ is denoted by $D_{y} f(x)$. In particular, for any function $t \mapsto v(t)$ defined on a real interval containing zero, we use the shorthand notation $v^{(i)}=\frac{\mathrm{d}^{i} v}{\mathrm{~d} t^{i}}(0)$ for all $i \in \mathbb{N}$.

Let $k \in \mathbb{N}$. The set of all $k$-jets from $\mathbb{R}^{n}$ to $\mathbb{R}^{m}$ is denoted by $J^{k}\left(\mathbb{R}^{n}, \mathbb{R}^{m}\right)$ (see, for instance, [11, Chapter II]). The mapping $\sigma: J_{x}^{k}\left(\mathbb{R}^{n}, \mathbb{R}^{m}\right) \rightarrow \mathbb{R}^{n}$ given by $\sigma: j^{k} f \mapsto \sigma\left(j^{k} f\right)=x$ is called the source map and the mapping $\tau: J_{x}^{k}\left(\mathbb{R}^{n}, \mathbb{R}^{m}\right) \rightarrow \mathbb{R}^{m}$ given by $\tau: j^{k} f \mapsto \tau\left(j^{k} f\right)=f(x)$ is called the target map. Put $J_{x}^{k}\left(\mathbb{R}^{n}, \mathbb{R}^{m}\right)=\sigma^{-1}(x), J^{k}\left(\mathbb{R}^{n}, \mathbb{R}^{m}\right)_{y}=\tau^{-1}(y)$ and $J_{x}^{k}\left(\mathbb{R}^{n}, \mathbb{R}^{m}\right)_{y}=$ $\sigma^{-1}(x) \cap \tau^{-1}(y)$. We have $J^{k}\left(\mathbb{R}^{n}, \mathbb{R}^{m}\right)=\coprod_{x \in \mathbb{R}^{n}} J_{x}^{k}\left(\mathbb{R}^{n}, \mathbb{R}^{m}\right)=\mathbb{R}^{n} \times J_{x}^{k}\left(\mathbb{R}^{n}, \mathbb{R}^{m}\right)$.

\section{Statement of the results}

\subsection{Problem statement}

Let $n$ be a positive integer, $A, B \in \operatorname{End}\left(\mathbb{R}^{n}\right), C \in \mathcal{L}\left(\mathbb{R}^{n}, \mathbb{R}\right), b \in \mathbb{R}^{n}$ and $u \in C^{\infty}\left(\mathbb{R}_{+}, \mathbb{R}\right)$. Set $A_{u}=A+u B$. In the present article, we focus on the following observed bilinear control system:

$$
\left\{\begin{array}{l}
\dot{x}=A_{u} x+b u \\
y=C x .
\end{array}\right.
$$

System (11) is said to be observable in time $T>0$ and for the control function $u$ if and only if, for all pair of solutions $\left(\left(x_{1}, y_{1}\right),\left(x_{2}, y_{2}\right)\right)$ of (11),$\left.\left(y_{1}-y_{2}\right)\right|_{[0, T]} \equiv 0$ implies $\left.\left(x_{1}-x_{2}\right)\right|_{[0, T]} \equiv 0$. For bilinear control systems of the form (11), we have the following characterization.

Proposition 2.1. System (11) is observable in time $T$ for the control $u$ if and only if for every $\omega_{0} \in \mathbb{S}^{n-1}$ the solution of $\dot{\omega}=A_{u(t)} \omega$ initiated from $\omega_{0}$ satisfies $\left.C \omega\right|_{[0, T]} \not \equiv 0$.

If (1) is observable for $u=0$ in some time $T>0$, then it is also observable in any time $T>0$, and we say that the pair $(C, A)$ is observable. According to the Kalman rank condition, $(C, A)$ is observable if and only if the rank of the following observability matrix

$$
\mathcal{O}(C, A)=\left(\begin{array}{c}
C \\
C A \\
\vdots \\
C A^{n-1}
\end{array}\right)
$$

is equal to $n$.

Let $\mathcal{S}$ be a finite dimensional manifold and let $L: \mathcal{S} \rightarrow \mathcal{L}\left(\mathbb{R}, \mathbb{R}^{n}\right)$. For all $u \in \mathbb{R}$, let $f(\cdot, u)$ be a vector field over $\mathcal{S}$. Denoting $\varepsilon=\hat{x}-x$, we introduce a dynamical observer system depending on the pair $(f, L)$ :

$$
\left\{\begin{array}{l}
\dot{\hat{x}}=A_{u} \hat{x}+b u-L(\xi) C \varepsilon \\
\dot{\varepsilon}=\left(A_{u}-L(\xi) C\right) \varepsilon \\
\dot{\xi}=f(\xi, u) .
\end{array}\right.
$$

Let $\lambda \in C^{\infty}\left(\mathbb{R}^{n}, \mathbb{R}\right)$ be such that 0 is an asymptotically stable equilibrium point of the vector field $x \mapsto A_{\lambda(x)} x+b \lambda(x)$ for some open domain of attraction $D(\lambda)$. We will further assume that $\lambda(0)=0$, which is true up to a substitution of $A$ with $A+\lambda(0) B$. 
As stated in the introduction, our goal is to make system (10) observable in time $T$ for the control $u=\lambda \circ \hat{x}$, where $\hat{x}$ follows (3) with initial conditions $\left(\hat{x}_{0}, \varepsilon_{0}, \xi_{0}\right)$. Since the stabilizing feedback $\lambda$ does not guarantee this property, we consider a small perturbation $\lambda+\delta$ of it. For all $\delta \in C^{\infty}\left(\mathbb{R}^{n}, \mathbb{R}\right)$, we consider the coupled system

$$
\left\{\begin{array}{l}
\dot{\hat{x}}=A_{(\lambda+\delta)(\hat{x})} \hat{x}+b(\lambda+\delta)(\hat{x})-L(\xi) C \varepsilon \\
\dot{\varepsilon}=\left(A_{(\lambda+\delta)(\hat{x})}-L(\xi) C\right) \varepsilon \\
\dot{\xi}=f(\xi,(\lambda+\delta)(\hat{x})) \\
\dot{\omega}=A_{(\lambda+\delta)(\hat{x})} \omega .
\end{array}\right.
$$

Remark 2.2. In system (44), the dynamics of $(\hat{x}, \varepsilon, \xi)$ do not depend on $\omega$. However, the dynamics of $\omega$ are included in (4) as they are crucial for the observability analysis of (11) with input $u=\lambda(\hat{x})$, as stated in Proposition 2.1. We will sometimes consider $(\hat{x}, \varepsilon, \xi)$ to be the first coordinates of a solution of (4) without fixing any initial condition for $\omega$.

From now on, we denote by $\mathcal{K}=\mathcal{K}_{1} \times \mathcal{K}_{2} \times \mathcal{K}_{3}$ a semi-algebraic compact subset of $D(\lambda) \times$ $\mathbb{R}^{n} \times \mathcal{S}$, which stands for a subset of the space of initial conditions of system (3). For all $R>0$, let

$$
\mathcal{V}_{R}=\left\{\delta \in C^{\infty}\left(\mathbb{R}^{n}, \mathbb{R}\right): \forall x \in B(0, R), \quad \delta(x)=0\right\} .
$$

We ask the observer given by $(f, L)$ to satisfy the following important properties:

(FC) (Forward completeness.) For all $u \in C^{\infty}\left(\mathbb{R}_{+}, \mathbb{R}\right)$, the time-varying vector field $f(\cdot, u)$ is forward complete. Moreover, for all $\left(\hat{x}_{0}, \varepsilon_{0}, \xi_{0}, \omega_{0}\right) \in \mathcal{K} \times \mathbb{S}^{n-1}$ and for all $\delta \in C^{\infty}\left(\mathbb{R}^{n}, \mathbb{R}\right)$ bounded over $D(\lambda)$, the coupled system (44) has a unique solution $(\hat{x}, \varepsilon, \xi, \omega) \in C^{\infty}\left(\mathbb{R}_{+}, \mathbb{R}^{n} \times \mathbb{R}^{n} \times \mathcal{S} \times \mathbb{S}^{n-1}\right)$ defined on $[0,+\infty)$.

(NFOT) (No flat observer trajectories.) For all $R>0$, there exists $\eta>0$ such that for all $\delta \in \mathcal{V}_{R}$ satisfying $\sup \left\{|\delta(x)|: x \in \mathcal{K}_{1}\right\}<\eta$, for all $\left(\hat{x}_{0}, \varepsilon_{0}, \xi_{0}, \omega_{0}\right) \in \mathcal{K} \times \mathbb{S}^{n-1}$ such that $\left(\hat{x}_{0}, \varepsilon_{0}\right) \neq(0,0)$, there exists a positive integer $k$ such that the solution of (4) with initial condition $\left(\hat{x}_{0}, \varepsilon_{0}, \xi_{0}, \omega_{0}\right)$ satisfies $\hat{x}^{(k)}(0) \neq 0$.

These properties are investigated in the last section of the paper. There, we show that the classical Luenberger and Kalman observers fit these hypotheses so that the main results may be applied to these observers.

For all $k \in \mathbb{N}, K \subset \mathbb{R}^{n}$ and $\delta \in C^{\infty}\left(\mathbb{R}^{n}, \mathbb{R}\right)$, let

$$
\|\delta\|_{k, K}=\sup \left\{\left|\frac{\partial^{\ell} \delta}{\partial x_{i_{1}} \cdots \partial x_{i_{\ell}}}(x)\right|: 0 \leqslant \ell \leqslant k, \quad 1 \leqslant i_{1} \leqslant \cdots \leqslant i_{\ell} \leqslant n, \quad x \in K\right\} .
$$

For any $k \in \mathbb{N}$, any compact subset $K \subset \mathbb{R}^{n}$ and any $\eta>0, k \in \mathbb{N}$, let

$$
\mathscr{N}(k, K, \eta)=\left\{\delta \in C^{\infty}\left(\mathbb{R}^{n}, \mathbb{R}\right):\|\delta\|_{k, K}<\eta\right\} .
$$

Remark 2.3. One can check that for any open subset $U \subset D(\lambda)$ relatively compact in $D(\lambda)$, for all $R>0$, there exists $\eta>0$ such that for all $\delta \in \mathcal{V}_{R}$ satisfying $|\delta|<\eta$, the feedback $\lambda+\delta$ is such that 0 is asymptotically stable with domain of attraction containing $U$. Hence in the following we focus only on the observability properties of the stabilizing feedback $\lambda+\delta$.

Main issue. Let $T>0$. Under genericity assumptions on $(A, B, C)$, does there exist $R, \eta>0$, a positive integer $k$ and a residual set $\mathscr{O} \subset \mathscr{N}\left(k, \mathcal{K}_{1}, \eta\right)$ such that we have the following property. For all $\delta \in \mathscr{O} \cap \mathcal{V}_{R}$ and for all initial conditions $\left(\hat{x}_{0}, \varepsilon_{0}, \xi_{0}\right) \in \mathcal{K}$, system (11) is observable in time $T$ for the control $u=(\lambda+\delta) \circ \hat{x}$, where $\hat{x}$ follows (44) with initial conditions $\left(\hat{x}_{0}, \varepsilon_{0}, \xi_{0}\right)$ and feedback perturbation $\delta$ ? 


\subsection{Main results}

In this section, we state the main results of the paper whose proofs are postponed to the upcoming sections. We first state our main theorem, that deals with the observability of system (4). Its proof is the most technical part of the paper, and heavily relies on transversality theory.

Theorem 2.4. Assume that the pairs $(C, A)$ and $(C, B)$ are observable. Assume that $0 \notin$ $\mathcal{K}_{1}$. Then there exist $\eta>0$, a positive integer $k$ and a dense open (in the Whitney $C^{\infty}$ topology) subset $\mathscr{O} \subset \mathscr{N}\left(k, \mathcal{K}_{1}, \eta\right)$ such that the solution to (4) with $\delta \in \mathscr{O}$ and initial condition $(\hat{x}(0), \varepsilon(0), \xi(0), \omega(0)) \in \mathcal{K} \times \mathbb{S}^{n-1}$ satisfies

$$
\exists k_{0} \in\{0, \ldots, k\} \quad:\left.\quad \frac{\mathrm{d}^{k_{0}}}{\mathrm{~d} t^{k_{0}}}\right|_{t=0} C \omega(t) \neq 0 .
$$

The proof of this theorem can be found in Section 3.2 .

Remark 2.5. Property (5) is stronger than observability of (4) in any time $T>0$. This implication is shown in Corollary 3.6. Pay attention to the assumption $0 \notin \mathcal{K}_{1}$. In Section 3.3. this assumption is removed, while only slightly weakening our observability result.

Theorem 2.4leads to the following corollary which states that under genericity assumptions on the system, there exists a generic class of perturbations $\delta$ such that the feedback $\lambda+\delta$ makes (4) observable.

Corollary 2.6. Assume that the pairs $(C, A)$ and $(C, B)$ are observable. Assume that 0 is in the interior of $\mathcal{K}_{1}$. Let $T>0$. Then there exist $R, \eta>0$, a positive integer $k$ and a dense open subset $\mathscr{O} \subset \mathscr{N}\left(k, \mathcal{K}_{1}, \eta\right) \cap \mathcal{V}_{R}$ such that the solution to (4) with $\delta \in \mathscr{O}$ and initial condition $\left(\hat{x}_{0}, \varepsilon_{0}, \xi_{0}, \omega_{0}\right) \in \mathcal{K} \times \mathbb{S}^{n-1}$ satisfies

$$
\exists t \in[0, T] \quad: \quad C \omega(t) \neq 0,
$$

that is system (11) is observable in time $T$ for the control $u=(\lambda+\delta) \circ \hat{x}$, where $\hat{x}$ follows (4) with initial conditions $\left(\hat{x}_{0}, \varepsilon_{0}, \xi_{0}\right)$ and feedback perturbation $\delta$.

This result also implies a generic observability property directly on the stabilizing state feedback law $\lambda$.

Corollary 2.7. Assume that the pairs $(C, A)$ and $(C, B)$ are observable. Assume that 0 is in the interior of $\mathcal{K}_{1}$. Denote by $\Lambda$ the set of feedbacks $\lambda \in C^{\infty}\left(\mathbb{R}^{n}, \mathbb{R}\right)$ such that 0 is a locally asymptotically stable equilibrium point of the vector field $x \mapsto A_{\lambda(x)} x+b \lambda(x)$. Let $T>0$ and $\Lambda_{T} \subset \Lambda$ be the set of feedbacks $\lambda \in \Lambda$ such that (10) is observable in time $T$ for the control $u=\lambda \circ \hat{x}$, where $\hat{x}$ follows (41) with $\delta \equiv 0$ and initial conditions $\left(\hat{x}_{0}, \varepsilon_{0}, \xi_{0}\right)$ in $\mathcal{K}$. Then $\Lambda_{T}$ is a dense open subset of $\Lambda$.

The proof of these two corollaries can be found in Section 3.3.

Remark 2.8. Because $\mathcal{V}_{R}$ is not open in the Whitney $C^{\infty}$ topology, the set $\mathscr{O}$ defined in Corollary [2.6] is not open in the Whitney $C^{\infty}$ topology, but it is open in the induced topology on $\mathscr{N}\left(k, \mathcal{K}_{1}, \eta\right) \cap \mathcal{V}_{R}$. Also, the set of matrices $(A, B, C) \in \operatorname{End}\left(\mathbb{R}^{n}\right)^{2} \times \mathcal{L}\left(\mathbb{R}^{n}, \mathbb{R}\right)$ such that $(C, A)$ and $(C, B)$ are both observable is open and dense. As a consequence, " $(C, A)$ and $(C, B)$ are observable" is a generic hypothesis. Contrarily to the strategy followed in [14] on some specific example, the results of this paper do not explicitly design any perturbation $\delta \in \mathscr{O}$, but rather state that for almost all bilinear system, almost all perturbation $\delta \in \mathscr{N}\left(k, \mathcal{K}_{1}, \eta\right) \cap \mathcal{V}_{R}$ belongs to $\mathscr{O}$ (in a topological sense). 
Finally, the next theorem shows that the classical Luenberger and Kalman observers fit hypotheses (FC) and (NFOT). Hence, our results may be applied to these well-known observers.

Theorem 2.9. Assume that $(C, A)$ is observable. Assume that $\lambda$ is bounded over $D(\lambda)$. Let $Q \in \mathcal{S}_{n}$. For all $\xi \in \mathcal{S}_{n}$ and all $u \in \mathbb{R}$, consider the following well-known observers:

$$
\begin{array}{lr}
f^{\text {Luenberger }}(\xi, u)=0 & \text { (Luenberger observer) } \\
f_{Q}^{\text {Kalman }}(\xi, u)=\xi A_{u}^{*}+A_{u} \xi+Q-\xi C^{*} C \xi & \text { (Kalman observer) }
\end{array}
$$

and $L(\xi)=\xi C^{*}$. Then the coupled system (4) given by $(f, L)$ satisfies the hypotheses (FC) and (NFOT) for any $f \in\left\{f^{\text {Luenberger }}, f_{Q}^{\text {Kalman }}\right\}$.

The proof of this theorem can be found in Section 4 ,

Remark 2.10. If $\lambda$ is unbounded over $D(\lambda)$, then for any open subset $U$ relatively compact in $D(\lambda)$, we can obtain by smooth saturation of $\lambda$ a new bounded feedback law $\lambda_{\text {sat }}$ such that $\lambda_{\text {sat } \mid U}=\lambda_{\mid U}$, for which the previous statement holds. (In particular $U \subset D\left(\lambda_{\text {sat }}\right)$.)

\section{Proofs of the observability statements}

In order to prove our main Theorem 2.4 and its Corollary 2.6. we need a series of preliminary results that we state and prove below. The main results will appear as corollaries of these subsequent lemmas.

Before we start the more technical elements of the paper, let us present the method we follow in order to prove the main results. Theorem 2.4 is an application of transversality theory to our particular problem (see [12] for the statements we rely on; see also [1, 11]). Consider a solution to (4) for a given perturbation $\delta$ of the feedback law, and a set of initial conditions in $\mathcal{K} \times \mathbb{S}^{n-1}$. We set $h: C^{\infty}\left(\mathbb{R}^{n}, \mathbb{R}\right) \times\left(\mathcal{K} \times \mathbb{S}^{n-1}\right) \times \mathbb{R}^{+} \rightarrow \mathbb{R}$ to be the smooth map given by

$$
h\left(\delta,\left(\hat{x}_{0}, \varepsilon_{0}, \xi_{0}, \omega_{0}\right), t\right)=C \omega(t) .
$$

As stated in Section 2, to get observability after perturbation of the feedback, we would like to show that there exists $\delta$, preferably small, such that

$$
t \mapsto h\left(\delta, z_{0}, t\right) \neq 0, \quad \forall z_{0}=\left(\hat{x}_{0}, \varepsilon_{0}, \xi_{0}, \omega_{0}\right) \in \mathcal{K} \times \mathbb{S}^{n-1} .
$$

A sufficient condition for $\delta$ to satisfy (6) is that for each $z_{0} \in \mathcal{K} \times \mathbb{S}^{n-1}$, there exists an integer $k$ such that $\left.\frac{\mathrm{d}^{k}}{\mathrm{~d} t^{k}}\right|_{t=0}\left(h\left(\delta, z_{0}, t\right)\right) \neq 0$. In other words, our goal will be achieved if we can prove that there exists $\delta$ and a finite set $\mathcal{I} \subset \mathbb{N}$ such that the map $H: C^{\infty}\left(\mathbb{R}^{n}, \mathbb{R}\right) \times\left(\mathcal{K} \times \mathbb{S}^{n-1}\right) \rightarrow \mathbb{R}^{|\mathcal{I}|}$ given by

$$
H\left(\delta, z_{0}\right)=\left(\left.\frac{\mathrm{d}^{k}}{\mathrm{~d} t^{k}}\right|_{t=0} h\left(\delta, z_{0}, t\right)\right)_{k \in \mathcal{I}},
$$

never vanishes. This is where transversality theory comes into play. Let $N$ denote the dimension of the surrounding space of $\mathcal{K} \times \mathbb{S}^{n-1}$. We can ensure that there exists $\delta$ satisfying (6) if we can prove that for some choice of $\mathcal{I}$, with $|\mathcal{I}|>N, H$ is transversal to $\{0\}$ at $\delta=0$. That is to say, if we can prove that the rank of the map $H(0, \cdot)$ is maximal, equal to $|\mathcal{I}|>N$, at any of its vanishing points (at which point $H(0, \cdot)$ is then a submersion).

Now it should be noted that in general, proving that a map is transversal to a point is a major hurdle, especially if the dimensions $n$ and $N$ of the spaces are unspecified. As a general rule, considering more orders of derivation of $h$ greatly increases the degrees of freedom of 
the map $H$ (by including higher order derivatives of $v$, as jet spaces grow exponentially in dimension), while only slightly increasing the size of the target space. This points towards an augmentation of the rank of $H$, making a proof of transversality achievable.

The difficulty lies however in producing a "rank increasing property" on $H$ as $|\mathcal{I}|$ increases. That is, finding a symmetry in the successive derivatives of $h$ that proves that for any dimension, a set $\mathcal{I}$ can be found by differentiating $h$ sufficiently many times.

The symmetry we use to prove the rank condition on the map $H$ can be described as follows. For $k \in \mathbb{N}$, let

$$
h^{k}\left(\delta, z_{0}, t\right)=C B^{k} \omega(t) .
$$

It turns out that if $h^{k+1}\left(0, z_{0}, \cdot\right)$ has a non-zero derivative of any order (including order 0 ), then we automatically get the rank condition for $h^{k}\left(0, z_{0}, \cdot\right)$ (this statement will be made precise in Corollary 3.31).

Here the hypothesis that $(C, B)$ is an observable pair becomes crucial. Indeed, observe that $h^{k}\left(0, z_{0}, 0\right)=C B^{k} \omega_{0}$. Hence, for any $\omega_{0} \in \mathbb{S}^{n-1}$ there exists a $k \in\{0, \ldots, n-1\}$ such that

$$
h^{k}\left(0, z_{0}, 0\right) \neq 0 \text {. }
$$

This in turns induces a partition of $\mathcal{K} \times \mathbb{S}^{n-1}$ into $n$ subsets on each of which at least one of the maps $h^{0}, \ldots, h^{n-1}$ never vanishes. Since $h^{k+1}\left(0, z_{0}, \cdot\right)$ not vanishing implies that the rank condition is satisfied for $h^{k}\left(0, z_{0}, \cdot\right)$, we chain-apply $n$ successive transversality theorems to prove the existence of a $\delta$ such that $h\left(\delta, z_{0}, \cdot\right)$ has always at least one non-zero time derivative at any point $z_{0} \in \mathcal{K} \times \mathbb{S}^{n-1}$.

Section 3.1 is aimed at making explicit the connection between the rank condition and the family of maps $\left(h^{k}\right)_{k \in \mathbb{N}}$. Section 3.2 is dedicated to the effective application of the principles presented in this introduction, which leads to the proof of Theorem 2.4. Section 3.3 concludes the proof of the observability statements by taking into account the behavior of the system near the target 0 .

\subsection{Preliminary results}

Let $u \in C^{\infty}\left(\mathbb{R}_{+}, \mathbb{R}\right)$ and consider the ordinary differential equation

$$
\dot{\omega}=(A+u(t) B) \omega .
$$

For all $k, m \in \mathbb{N}$, let $F_{k}^{m}: C^{\infty}\left(\mathbb{R}_{+}, \mathbb{R}\right) \times \mathbb{R}^{n} \rightarrow \mathbb{R}$ be the function such that

$$
F_{k}^{m}\left(u, \omega_{0}\right)=C B^{m} \omega^{(k)}(0)
$$

where $t \mapsto \omega(t)$ is the solution of (7) with initial condition $\omega_{0}$.

Let us introduce the $n \times n$ matrix valued polynomials in the indeterminates $X_{0}, \ldots, X_{k-1}$ by:

$$
\operatorname{End}\left(\mathbb{R}^{n}\right)\left[X_{0}, \ldots X_{k-1}\right]= \begin{cases}\operatorname{End}\left(\mathbb{R}^{n}\right) & \text { if } k=0 \\ \operatorname{End}\left(\mathbb{R}^{n}\right)\left[X_{0}, \ldots X_{k-2}\right]\left[X_{k-1}\right] & \text { otherwise }\end{cases}
$$

and set

$$
\operatorname{End}\left(\mathbb{R}^{n}\right)\left[\left(X_{k}\right)_{k \in \mathbb{N}}\right]=\bigcup_{k \in \mathbb{N}} \operatorname{End}\left(\mathbb{R}^{n}\right)\left[X_{0}, \ldots X_{k-1}\right] .
$$

Let $\Psi: \operatorname{End}\left(\mathbb{R}^{n}\right)\left[\left(X_{k}\right)_{k \in \mathbb{N}}\right] \rightarrow \operatorname{End}\left(\mathbb{R}^{n}\right)\left[\left(X_{k}\right)_{k \in \mathbb{N}}\right]$ be the linear map defined by

$$
\Psi(P)\left(X_{0}, \ldots, X_{k}\right)=P\left(X_{0}, \ldots, X_{k-1}\right)\left(A+X_{0} B\right)+\sum_{i=0}^{k-1} \frac{\partial P}{\partial X_{i}}\left(X_{0}, \ldots, X_{k-1}\right) X_{i+1},
$$


where $k=\min \left\{\ell \in \mathbb{N}: P \in \operatorname{End}\left(\mathbb{R}^{n}\right)\left[X_{0}, \ldots X_{\ell-1}\right]\right\}$.

Finally, let us define the family $\left(P_{k}\right)_{k \in \mathbb{N}}$ of matrix valued polynomials such that $P_{0} \in$ $\operatorname{End}\left(\mathbb{R}^{n}\right)$ and $P_{k} \in \operatorname{End}\left(\mathbb{R}^{n}\right)\left[X_{0}, \ldots X_{k-1}\right]$, for all $k \geqslant 1$, by

$$
P_{0}=\mathrm{I}, \quad P_{k+1}=\Psi\left(P_{k}\right), \quad \forall k \in \mathbb{N} .
$$

It is clear 1 that for all $m \in \mathbb{N}$,

$$
F_{k}^{m}\left(u, \omega_{0}\right)= \begin{cases}C B^{m} \omega_{0} & \text { if } k=0 \\ C B^{m} P_{k}\left(u^{(0)}, u^{(1)}, \ldots, u^{(k-1)}\right) \omega_{0} & \text { otherwise }\end{cases}
$$

where $u^{(i)}$ is shorthand for $\frac{\mathrm{d}^{i} u}{\mathrm{~d} t^{i}}(0)$ for all $i \in \mathbb{N}$. For all $k \in \mathbb{N}$ and $i \in \mathbb{N}, 1 \leqslant i \leqslant k$, let $Q_{i}^{k}=\frac{\partial P_{k}}{\partial X_{k-i}}$.

Lemma 3.1. For all $i \in \mathbb{N} \backslash\{0\}$, there exist $R_{i}^{0}, \ldots, R_{i}^{i-1} \in \operatorname{End}\left(\mathbb{R}^{n}\right)\left[X_{0}, \ldots X_{i-1}\right]$ such that 2

$$
Q_{i}^{i+k}=\sum_{j=0}^{i-1} k^{j} R_{i}^{j}, \quad \forall k \geqslant 0 .
$$

Furthermore, $R_{i}^{i-1}=\frac{B P_{i-1}}{(i-1) !}$.

Proof. We prove the first part of the statement by induction on $i$. For $i=1$, one easily checks that

$$
Q_{1}^{1+k}=B, \quad \forall k \in \mathbb{N} .
$$

Assuming the desired property for $i$, we have to prove that there exist $R_{i+1}^{0}, \ldots, R_{i+1}^{i} \in$ $\operatorname{End}\left(\mathbb{R}^{n}\right)\left[X_{0}, \ldots X_{i}\right]$ such that

$$
Q_{i+1}^{i+1+k}=\sum_{j=0}^{i} k^{j} R_{i+1}^{j}, \quad \forall k \geqslant 0 .
$$

Using the definition of $Q_{i+1}^{i+1+\ell}$ and the recurrence relation (8) yields

$$
Q_{i+1}^{i+1+\ell}=\Psi\left(Q_{i}^{i+\ell}\right)+Q_{i+1}^{i+\ell}, \quad \forall \ell \geqslant 1 .
$$

\footnotetext{
${ }^{1}$ Note that, for $k \neq 0$, the function $F_{k}^{m}$ actually acts on $(k-1)$-jets at zero of functions and not on functions themselves. Consequently, the restriction $\left.F_{k}^{m}\right|_{J_{0}^{\ell}(\mathbb{R}, \mathbb{R}) \times \mathbb{R}^{n}}$ is well-defined as soon as $\ell \geqslant k-1$. Of course, for $k=0$, the restriction $\left.F_{0}^{m}\right|_{J_{0}^{\ell}(\mathbb{R}, \mathbb{R}) \times \mathbb{R}^{n}}$ makes sense only if $\ell \geqslant 0$. In summary, the restriction $\left.F_{k}^{m}\right|_{J_{0}^{\ell}(\mathbb{R}, \mathbb{R}) \times \mathbb{R}^{n}}$ is well-defined as soon as $\ell \geqslant k$.

${ }^{2}$ Actually, we can show that $R_{i}^{0}, \ldots, R_{i}^{i-1} \in \operatorname{End}\left(\mathbb{R}^{n}\right)\left[X_{0}, \ldots X_{i-2}\right]$
} 
Consequently, for all $k \geqslant 0$,

$$
\begin{aligned}
Q_{i+1}^{i+1+k}= & \sum_{\ell=1}^{k}\left(Q_{i+1}^{i+1+\ell}-Q_{i+1}^{i+\ell}\right)+Q_{i+1}^{i+1} \\
= & \sum_{\ell=1}^{k}\left(\Psi\left(Q_{i}^{i+\ell}\right)\right)+Q_{i+1}^{i+1} \\
= & \left.\sum_{\ell=1}^{k}\left(\sum_{j=0}^{i-1} \ell^{j} \Psi\left(R_{i}^{j}\right)\right)+Q_{i+1}^{i+1} \quad \quad \text { (by (10) }\right) \\
= & \sum_{j=0}^{i-1}\left(\sum_{\ell=1}^{k} \ell^{j}\right) \Psi\left(R_{i}^{j}\right)+Q_{i+1}^{i+1} \\
= & \sum_{j=0}^{i-1} S^{j}(k) \Psi\left(R_{i}^{j}\right)+Q_{i+1}^{i+1}, \quad \text { with } S^{j}(k)=\sum_{\ell=1}^{k} \ell^{j} .
\end{aligned}
$$

Note that $Q_{i+1}^{i+1}, \Psi\left(R_{i}^{j}\right) \in \operatorname{End}\left(\mathbb{R}^{n}\right)\left[X_{0}, \ldots, X_{i}\right]$ for all $j \in\{0, \ldots, i-1\}\left(Q_{i+1}^{i+1}=\partial P_{i+1} / \partial X_{0}\right)$. Moreover, according to Faulhaber's formula, we have

$$
S^{j}(k)=\frac{k^{j+1}}{j+1}+T^{j}(k), \quad \forall j, k \in \mathbb{N},
$$

where $T^{j}(k)$ is a polynomial in the variable $k$ of degree $j$ with no constant term. Consequently,

$$
\begin{aligned}
Q_{i+1}^{i+1+k} & =\frac{k^{i}}{i} \Psi\left(R_{i}^{i-1}\right)+\left(T^{i-1}(k) \Psi\left(R_{i}^{i-1}\right)+\sum_{j=0}^{i-2} S^{j}(k) \Psi\left(R_{i}^{j}\right)\right)+Q_{i+1}^{i+1} \\
& =k^{i} R_{i+1}^{i}+\sum_{j=1}^{i-1} k^{j} R_{i+1}^{j}+R_{i+1}^{0} \\
& =\sum_{j=0}^{i} k^{j} R_{i+1}^{j},
\end{aligned}
$$

with $R_{i+1}^{i}=\Psi\left(R_{i}^{i-1}\right) / i, R_{i+1}^{0}=Q_{i+1}^{i+1}$ and $R_{i+1}^{j} \in \operatorname{End}\left(\mathbb{R}^{n}\right)\left[X_{0}, \ldots, X_{i}\right]$ for all $j \in\{0, \ldots, i\}$.

The second part of the statement easily follows by induction. Indeed,

$$
B P_{0}=Q_{1}^{1}=\sum_{j=0}^{0} 0^{j} R_{1}^{j}=R_{1}^{0},
$$

and

$$
R_{i+1}^{i}=\frac{\Psi\left(R_{i}^{i-1}\right)}{i}=\frac{1}{i} \Psi\left(\frac{1}{(i-1) !} B P_{i-1}\right)=\frac{1}{i !} B \Psi\left(P_{i-1}\right)=\frac{1}{i !} B P_{i} .
$$

The statement follows.

Corollary 3.2. Let $i, m \in \mathbb{N}, i \geqslant 1$. Let $v \in \mathbb{R}^{i}$ and $\omega_{0} \in \mathbb{R}^{n}$. Either there exists $k_{0} \geqslant i$ such that $C B^{m} Q_{i}^{k}(v) \omega_{0} \neq 0$ for all $k \geqslant k_{0}$ or $C B^{m} Q_{i}^{k}(v) \omega_{0}=0$ for all $k \geqslant i$.

Proof. By Lemma 3.1, we have $Q_{i}^{k}=\sum_{j=0}^{i-1}(k-i)^{j} R_{i}^{j}$ for all integer $k \geqslant i$. If $C B^{m} R_{i}^{j}(v) \omega_{0}=0$ for all $j \in\{0, \ldots, i-1\}$, then $C B^{m} Q_{i}^{k}(v) \omega_{0}=0$ for all $k \geqslant i$. Otherwise, there exists 
$j \in\{0, \ldots, i-1\}$ such that $C B^{m} R_{i}^{j}(v) \omega_{0} \neq 0$. Let $\left(k_{0}, \ldots k_{i-1}\right) \in \mathbb{N}^{i}$ with $k_{0}<\cdots<k_{i-1}$. We have

$$
C B^{m}\left(\begin{array}{c}
Q_{i}^{i+k_{0}}(v) \\
\vdots \\
Q_{i}^{i+k_{i-1}(v)}
\end{array}\right) \omega_{0}=\left(\begin{array}{cccc}
1 & k_{0} & \ldots & k_{0}^{i} \\
\vdots & \vdots & & \vdots \\
1 & k_{i-1} & \ldots & k_{i-1}^{i}
\end{array}\right) C B^{m}\left(\begin{array}{c}
R_{i}^{0}(v) \\
\vdots \\
R_{i}^{i-1}(v)
\end{array}\right) \omega_{0} .
$$

Since $k_{0}, \ldots k_{i-1}$ are pairwise different, the Vandermonde matrix is invertible. Consequently, there exits $j \in\{0, \ldots, i-1\}$ such that $C B^{m} Q_{i}^{i+k_{j}}(v) \omega_{0} \neq 0$. Hence, there exists at most $i-1$ positive integers $k_{j}$ such that $C B^{m} Q_{i}^{i+k_{j}}(v) \omega_{0}=0$. Thus, there exists $k_{0} \geqslant i$ such that $C B^{m} Q_{i}^{k}(v) \omega_{0} \neq 0$ for all $k \geqslant k_{0}$.

For all $P \in \operatorname{End}\left(\mathbb{R}^{n}\right)\left[X_{0}, \ldots X_{k-1}\right]$ and all $v \in \mathbb{R}^{\mathbb{N}}$, we set $P(v)=P\left(v_{0}, \ldots, v_{k-1}\right)$.

Corollary 3.3. Let $v \in \mathbb{R}^{\mathbb{N}}, \omega_{0} \in \mathbb{R}^{n}$ and $m \in \mathbb{N}$. If there exists $i \in \mathbb{N} \backslash\{0\}$ such that $C B^{m+1} P_{i-1}(v) \omega_{0} \neq 0$, then there exists $k_{0} \in \mathbb{N}$ such that, for all $N \in \mathbb{N} \backslash\{0\}$, the mapping 3 $\varphi: J_{0}^{k_{0}+N-1}(\mathbb{R}, \mathbb{R})=\mathbb{R}^{k_{0}+N} \rightarrow \mathbb{R}^{N}$ defined by

$$
\varphi(\cdot)=\left(C B^{m} P_{k_{0}}(\cdot) \omega_{0}, \ldots, C B^{m} P_{k_{0}+N-1}(\cdot) \omega_{0}\right)
$$

has a rank $N$ differential at $\left(v_{0}, \ldots, v_{k_{0}+N-1}\right)$.

Proof. Assume that there exists $i \geqslant 1$ such that $C B^{m+1} P_{i-1}(v) \omega_{0} \neq 0$. Since, according to Lemma 3.1, $R_{i}^{i-1}=B P_{i-1} /(i-1)$ !, this is equivalent to $C B^{m} R_{i}^{i-1}(v) \omega_{0} \neq 0$. Thus, reasoning as in the proof of Corollary 3.2 , the sequence $\left(C B^{m} Q_{i}^{k}(v) \omega_{0}\right)_{k \geqslant i}$ is not constant equal to zero. Set

$$
i_{0}=\min \left\{i \in \mathbb{N} \backslash\{0\}:\left(C B^{m} Q_{i}^{k}(v) \omega_{0}\right)_{k \geqslant i} \not \equiv 0\right\} .
$$

As a consequence of Corollary [3.2, there exists $k_{0} \in \mathbb{N}$ such that $C B^{m} Q_{i_{0}}^{k}(v) \omega_{0} \neq 0$ for all $k \geqslant k_{0}$, i.e.

$$
\frac{\partial\left(C B^{m} P_{k} \omega_{0}\right)}{\partial X_{k-i_{0}}}\left(v_{0}, \ldots, v_{k_{0}+N-1}\right)=\frac{\partial\left(C B^{m} P_{k} \omega_{0}\right)}{\partial X_{k-i_{0}}}(v) \neq 0, \quad \forall k \geqslant k_{0},
$$

and (by construction of $i_{0}$ )

$$
\frac{\partial\left(C B^{m} P_{k} \omega_{0}\right)}{\partial X_{\ell}}\left(v_{0}, \ldots, v_{k_{0}+N-1}\right)=\frac{\partial\left(C B^{m} P_{k} \omega_{0}\right)}{\partial X_{\ell}}(v)=0, \quad \forall \ell>k-i_{0} .
$$

In other words,

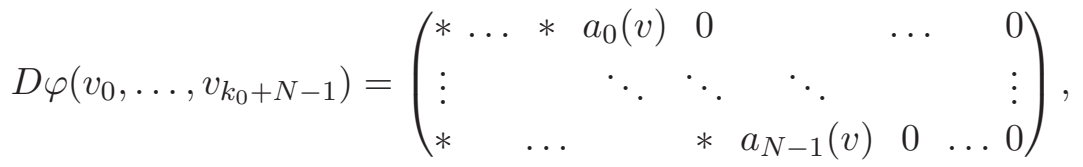

with $a_{i}(v)=C B^{m} Q_{i_{0}}^{k_{0}+i}(v) \omega_{0}$. The statement follows.

\footnotetext{
${ }^{3}$ Note that $\varphi(\cdot)=F_{\left\{k_{0}, \ldots, k_{0}+N-1\right\}}^{m}\left(\cdot, \omega_{0}\right)$, with $F_{\left\{k_{0}, \ldots, k_{0}+N-1\right\}}^{m}$ defined as in Section 3.2
} 


\subsection{Observability away from the target and proof of Theorem 2.4}

Using the results of the previous section, we are now able to prove our main Theorem 2.4 , In this section, we assume that $0 \notin \mathcal{K}_{1}$. From now on $t \mapsto(\hat{x}(t), \varepsilon(t), \xi(t), \omega(t))$, or simply $(\hat{x}, \varepsilon, \xi, \omega)$, denotes the solution to (4) with initial condition $\left(\hat{x}_{0}, \varepsilon_{0}, \xi_{0}, \omega_{0}\right)$.

Let us introduce some new notation. For any $k \in \mathbb{N}$, define the map $G^{k}$ by:

$$
\begin{aligned}
G^{k}: J^{k}\left(\mathbb{R}^{n}, \mathbb{R}\right) \times \mathcal{K}_{2} \times \mathcal{K}_{3} & \longrightarrow J_{0}^{k}(\mathbb{R}, \mathbb{R}) \\
\left(j^{k} \delta\left(\hat{x}_{0}\right), \varepsilon_{0}, \xi_{0}\right) & \longmapsto j^{k}((\lambda+\delta) \circ \hat{x})(0) .
\end{aligned}
$$

For any finite subset $\mathcal{I} \subset \mathbb{N}$ and any $m \in \mathbb{N}$, set $k_{\mathcal{I}}=\max \mathcal{I}$ and define the maps, $F_{\mathcal{I}}^{m}$ and $H_{\mathcal{I}}^{m}$ as follows:

$$
\begin{aligned}
& F_{\mathcal{I}}^{m}: J_{0}^{k_{\mathcal{I}}}(\mathbb{R}, \mathbb{R}) \times \mathbb{S}^{n-1} \longrightarrow \mathbb{R}^{|\mathcal{I}|}\left(v, \omega_{0}\right) \longmapsto\left(C B^{m} P_{k}(v) \omega_{0}\right)_{k \in \mathcal{I}}, \\
& H_{\mathcal{I}}^{m}=F_{\mathcal{I}}^{m} \circ\left(G^{k_{\mathcal{I}}} \times \mathrm{I}_{\mathbb{S}^{n-1}}\right) .
\end{aligned}
$$

Remark 3.4. Notice that for any $m, k_{0} \in \mathbb{N}$ and any $N \in \mathbb{N} \backslash\{0\}$ such that $\mathcal{I} \subset\left\{k_{0}, \ldots, k_{0}+\right.$ $N-1\}$, the map $F_{\mathcal{I}}^{m}$ satisfies

$$
F_{\mathcal{I}}^{m}=\pi_{\mathcal{I}} \circ F_{\left\{k_{0}, \ldots, k_{0}+N-1\right\}}^{m},
$$

where $\pi_{\mathcal{I}}: J_{0}^{k_{0}+N-1}(\mathbb{R}, \mathbb{R})=\mathbb{R}^{k_{0}+N} \rightarrow \mathbb{R}^{|\mathcal{I}|}$ denotes the canonical projection onto the factors that correspond to indices in $\mathcal{I}$.

Now we state the following proposition, which leads directly to Theorem 2.4

Proposition 3.5. For all $m \in\{0, \ldots, n-1\}$, define

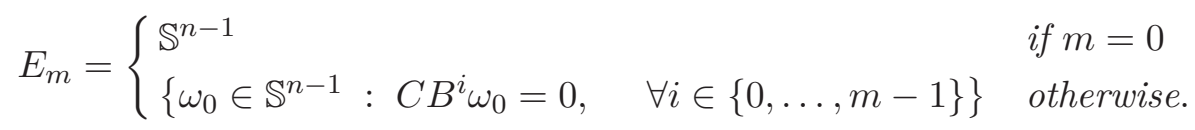

Suppose $(C, A)$ and $(C, B)$ are observable pairs. Then for every $m \in\{0, \ldots, n-1\}$, there exist $k \in \mathbb{N}$, a positive real number $\eta$ and a dense open subset $\mathscr{O}_{m} \subset \mathscr{N}\left(k, \mathcal{K}_{1}, \eta\right)$ such that for all $\left(\delta, \hat{x}_{0}, \varepsilon_{0}, \xi_{0}, \omega_{0}\right) \in \mathscr{O}_{m} \times \mathcal{K} \times E_{m}$

$$
H_{\{0, \ldots, k\}}^{m}\left(j^{k} \delta\left(\hat{x}_{0}\right), \varepsilon_{0}, \xi_{0}, \omega_{0}\right) \neq 0 .
$$

Proof. The proof strongly relies on the results of Section 3.1 and on the Goresky-MacPherson transversality theorem (see [12, Part I, Chapter 1]). We prove the proposition by finite descending induction on $m$. Note that since the pair $(C, B)$ is observable, we have $\varnothing=E_{n} \subset$ $E_{n-1} \subset \cdots \subset E_{1} \varsubsetneqq E_{0}=\mathbb{S}^{n-1}$.

For $m=n-1$, the result is immediate because, by observability of the pair $(C, B)$, $C B^{n-1} \omega_{0} \neq 0$ for all $\omega_{0} \in E_{n-1}$. Hence, for $k=0$ and any positive real number $\eta$, we have for all $\left(\delta, \hat{x}_{0}, \varepsilon_{0}, \xi_{0}, \omega_{0}\right) \in \mathscr{N}\left(k, \mathcal{K}_{1}, \eta\right) \times \mathcal{K} \times E_{n-1}$,

$$
H_{\{0\}}^{n-1}\left(j^{0} \delta\left(\hat{x}_{0}\right), \varepsilon_{0}, \xi_{0}, \omega_{0}\right)=C B^{n-1} \omega_{0} \neq 0 .
$$

Now suppose $1 \leqslant m \leqslant n-1$. Note that, by definition of $E_{m-1} \backslash E_{m}$,

$$
C B^{m-1} \omega_{0} \neq 0, \quad \forall \omega_{0} \in E_{m-1} \backslash E_{m} .
$$


Assume that we are given a $k \in \mathbb{N}$, a positive real number $\eta$ and a dense open subset $\mathscr{O}_{m} \subset \mathscr{N}\left(k, \mathcal{K}_{1}, \eta\right)$ such that

$$
H_{\{0, \ldots, k\}}^{m}\left(j^{k} \delta\left(\hat{x}_{0}\right), \varepsilon_{0}, \xi_{0}, \omega_{0}\right) \neq 0, \quad \forall\left(\delta, \hat{x}_{0}, \varepsilon_{0}, \xi_{0}, \omega_{0}\right) \in \mathscr{O}_{m} \times \mathcal{K} \times E_{m} .
$$

Choose $\left(\delta, \hat{x}_{0}, \varepsilon_{0}, \xi_{0}, \omega_{0}\right) \in \mathscr{O}_{m} \times \mathcal{K} \times E_{m}$ and put $u(t)=(\lambda+\delta)(\hat{x}(t))$. Equation (14) implies that $C B^{m} P_{i}\left(u^{(0)}, \ldots, u^{(k)}\right) \omega_{0} \neq 0$ for an integer $i \in\{0, \ldots, k\}$, so, by Corollary 3.3 there exists $k_{0} \in \mathbb{N}$ such that, for any positive integer $k_{1}$, the map $F_{\left\{k_{0}, \ldots, k_{0}+k_{1}-1\right\}}^{m-1}$ has a rank $k_{1}$ differential at $\left(u^{(0)}, \ldots, u^{\left(k_{0}+k_{1}-1\right)}\right)$.

Let $i_{0} \in \mathbb{N}$ be defined as in the proof of Corollary 3.3. Let $p \in \mathbb{N} \backslash\{0\}$ be such that $\hat{x}^{(p)} \neq 0$ and $\hat{x}^{(q)}=0$ for all $q<p$ (which exists by hypothesis (NFOT) and $0 \notin \mathcal{K}_{1}$ ), and choose $\ell \in\{1, \ldots n\}$ so that $\hat{x}_{\ell}^{(p)} \neq 0$. Put

$$
j_{0}=\min \left\{j \geqslant k_{0}: j-i_{0} \equiv 0 \quad(\bmod p)\right\} \text { 约 and } \mathcal{I}=\left\{j_{0}+r p: r \in\{0, \ldots, N-1\}\right\},
$$

where $N$ is a positive integer. The (partial) differential of $G_{\mathcal{I}}^{m}$ with respect to

$$
w=\left.\left(\delta, \frac{\partial}{\partial x_{\ell}} \delta, \ldots,\left(\frac{\partial}{\partial x_{\ell}}\right)^{k_{\mathcal{I}}} \delta\right)\right|_{x=\hat{x}_{0}}
$$

at $X_{0}=\left(j^{k_{\mathcal{I}}} \delta\left(\hat{x}_{0}\right), \varepsilon_{0}, \xi_{0}, \omega_{0}\right)$ is the submatrix $D_{w} G_{\mathcal{I}}^{m}\left(X_{0}\right)$ obtained from $D G_{\mathcal{I}}^{m}\left(X_{0}\right)$ by deleting all columns that do not correspond to partial derivatives with respect to $w$. In other words,

$$
D_{w} G_{\mathcal{I}}^{m}\left(X_{0}\right)=\left(\operatorname{col}(0) \cdots \operatorname{col}\left(k_{\mathcal{I}}-1\right)\right) .
$$

Each column $\operatorname{col}(i), i \in\left\{0, \ldots, k_{\mathcal{I}}-1\right\}$ of $D_{w} G_{\mathcal{I}}^{m}\left(X_{0}\right)$ satisfies

$$
\operatorname{col}(i)^{*}=\left(\begin{array}{llll}
0 & \cdots & 0 & \left.b_{i}\left(X_{0}\right) * \cdots *\right)^{*}, \quad b_{i}\left(X_{0}\right) \neq 0,
\end{array}\right.
$$

where the non zero coefficient $b_{i}\left(X_{0}\right)$ appears at the $i p$ th row. According to Faà di Bruno formula, we have

$$
b_{i}\left(X_{0}\right)=n_{i}\left(\hat{x}_{\ell}^{(p)}\right)^{i}
$$

$n_{i}$ being a positive integer for each $i \in\left\{0, \ldots, k_{\mathcal{I}}-1\right\}$.

It is clear from the definition of $F_{\mathcal{I}}^{m}$ and Remark 3.4 thereafter that $D F_{\mathcal{I}}^{m}$ is the submatrix of $D F_{\left\{k_{0}, \ldots, k_{\mathcal{I}}\right\}}^{m}$ (see equation (12) ) obtained by keeping the $i$ th rows for $i \in \mathcal{I}$. Therefore,

$$
\begin{aligned}
\operatorname{rank}\left(D H_{\mathcal{I}}^{m}\left(X_{0}\right)\right) & \geqslant \operatorname{rank}\left(D_{v} F_{\mathcal{I}}^{m}\left(G^{k_{\mathcal{I}}}\left(X_{0}\right), \omega_{0}\right) \circ D_{w} G^{k_{\mathcal{I}}}\left(X_{0}\right)\right) \\
& =\operatorname{rank}\left(\begin{array}{cccccccc}
* \cdots & * & c_{0}\left(X_{0}\right) & 0 & & \cdots & 0 \\
\vdots & & \ddots & \ddots & \ddots & & \vdots \\
* & \ldots & & * & c_{N-1}\left(X_{0}\right) & 0 & \cdots & 0
\end{array}\right),
\end{aligned}
$$

where $c_{r}\left(X_{0}\right)=a_{j_{0}+r p}\left(G^{k_{\mathcal{I}}}\left(X_{0}\right), \omega_{0}\right) b_{j_{0}+r p}\left(X_{0}\right), r \in\{0, \ldots, N-1\}$. Hence $H_{\mathcal{I}}^{m-1}$ has a rank $N$ differential at $X_{0}$.

For any $k \in \mathbb{N}$, any compact subset $K \subset \mathbb{R}^{n}$ and any $\eta>0, k \in \mathbb{N}$, define

$$
\mathcal{M}(k, K, \eta)=\left\{\alpha \in J^{k}\left(\mathbb{R}^{n}, \mathbb{R}\right): \exists f \in \mathscr{N}(k, K, \eta), \quad \exists a \in K, \quad \alpha=j^{k} f(a)\right\} .
$$

\footnotetext{
${ }^{4}$ Index $j_{0}$ corresponds to the smallest index $j \geqslant k_{0}$ such that $\hat{x}_{\ell}^{(p)}$ appears in $u^{\left(j-i_{0}\right)}$.
} 
Clearly, $\mathcal{M}(k, K, \eta)$ is an open submanifold of $J^{k}\left(\mathbb{R}^{n}, \mathbb{R}\right)$.

Since the rank is a semi-continuous map, there exists a neighborhood $V \subset \mathcal{M}\left(k_{\mathcal{I}}, \mathcal{K}_{1}, \eta\right) \times$ $\mathcal{K}_{2} \times \mathcal{K}_{3} \times E_{m}$ of $\left(j_{0}^{k_{\mathcal{I}}}\left(\hat{x}_{0}\right), \varepsilon_{0}, \xi_{0}, \omega_{0}\right)$ such that $H_{\mathcal{I}}^{m-1}$ has a rank $N$ on $V$. Let $\rho \in(0, \eta)$ and $\mathcal{C}(\rho)=\mathcal{C}_{1} \times \mathcal{C}_{2} \times \mathcal{C}_{3} \times \Omega_{m}$ be a semi-algebraic compact subset of $\mathcal{K} \times E_{m}$ such that

$$
W:=\mathcal{M}\left(k_{\mathcal{I}}, \mathcal{K}_{1}, \rho\right) \times \mathcal{C}_{2} \times \mathcal{C}_{3} \times \Omega_{m} \subset V .
$$

Let $B=\left(\left.H_{\mathcal{I}}^{m-1}\right|_{W}\right)^{-1}(0)$ and $Z=\pi(B)$, where $\pi$ is the projection that is parallel to $\mathcal{C}_{2} \times$ $\mathcal{C}_{3} \times \Omega_{m}$. Then, and because $\mathcal{C}_{2} \times \mathcal{C}_{3} \times \Omega_{m}$ is compact, $Z \subset \mathcal{M}\left(k_{\mathcal{I}}, \mathcal{K}_{1}, \rho\right)$ is a closed semialgebraic subset. Hence, according to the Goresky-McPherson transversality theorem ([12, Part I, Chapter 1, page 38, Proposition]), the set

$$
\tilde{\mathscr{O}}(\rho)=\left\{f \in C^{\infty}\left(\mathbb{R}^{n}, \mathcal{M}\left(k_{\mathcal{I}}, \mathcal{K}_{1}, \rho\right)\right):\left.f\right|_{\mathcal{C}_{1}} \text { is transversal to } Z\right\}
$$

is open and dense (in the Whitney $C^{\infty}$ topology) in $C^{\infty}\left(\mathbb{R}^{n}, \mathcal{M}\left(k_{\mathcal{I}}, \mathcal{K}_{1}, \rho\right)\right)$. Moreover, since $\left.H_{\mathcal{I}}^{m-1}\right|_{W}$ is a submersion, we have $\operatorname{codim}_{\mathcal{M}\left(k_{\mathcal{I}}, \mathcal{K}_{1}, \rho\right)} Z \geqslant \operatorname{codim}_{\mathbb{R}^{N}}\{0\}-\operatorname{dim}\left(\mathcal{C}(\rho) \times E_{m}\right)=$ $N-\operatorname{dim}\left(\mathcal{C}(\rho) \times E_{m}\right)$. Picking $N$ sufficiently large, we have

$$
\operatorname{codim}_{\mathcal{M}\left(k_{\mathcal{I}}, \mathcal{K}_{1}, \rho\right)} Z>n
$$

in which case, transversal necessarily means to avoid. It follows that

$$
\begin{aligned}
\tilde{\mathscr{O}}(\rho) & =\left\{f \in C^{\infty}\left(\mathbb{R}^{n}, \mathcal{M}\left(k_{\mathcal{I}}, \mathcal{K}_{1}, \rho\right)\right): \forall \hat{x} \in \mathcal{C}_{1}, f(\hat{x}) \notin Z\right\} \\
& =\left\{f \in C^{\infty}\left(\mathbb{R}^{n}, \mathcal{M}\left(k_{\mathcal{I}}, \mathcal{K}_{1}, \rho\right)\right): \forall(\hat{x}, \varepsilon, \xi, \omega) \in \mathcal{C}(\rho),(f(\hat{x}), \varepsilon, \xi, \omega) \notin B\right\} \\
& =\left\{f \in C^{\infty}\left(\mathbb{R}^{n}, \mathcal{M}\left(k_{\mathcal{I}}, \mathcal{K}_{1}, \rho\right)\right): \forall(\hat{x}, \varepsilon, \xi, \omega) \in \mathcal{C}(\rho), H_{\mathcal{I}}^{m-1}(f(\hat{x}), \varepsilon, \xi, \omega) \neq 0\right\} .
\end{aligned}
$$

By compactness of $\mathcal{K} \times E_{m}$, there exists $q \in \mathbb{N}$ such that

$$
\mathcal{K} \times E_{m}=\bigcup_{i=1}^{q} \mathcal{C}\left(\rho_{i}\right)
$$

Set $\eta=\min \left\{\rho_{i} \quad: \quad i=1, \ldots, q\right\}>0, k=\max \left\{k_{\mathcal{I}}\left(\rho_{i}\right) \quad: \quad i=1, \ldots, q\right\}$ and define $\tilde{\mathscr{O}}=$ $\bigcap_{i=1}^{q} \tilde{\mathscr{O}}\left(\rho_{i}\right)$. According to (15),

$$
\begin{aligned}
& \tilde{\mathscr{O}}=\left\{f \in C^{\infty}\left(\mathbb{R}^{n}, \mathcal{M}\left(k, \mathcal{K}_{1}, \eta\right)\right): \forall(\hat{x}, \varepsilon, \xi, \omega) \in \mathcal{K} \times E_{m},\right. \\
& \left.H_{\{0, \ldots, k\}}^{m-1}(f(\hat{x}), \varepsilon, \xi, \omega) \neq 0\right\} .
\end{aligned}
$$

Also, by definition of $E_{m-1}$ and $E_{m}, H_{\{0\}}^{m-1}(\omega)=C B^{m-1} \omega \neq 0$ for all $\omega \in E_{m-1} \backslash E_{m}$. Thus,

$$
\begin{aligned}
& \tilde{\mathscr{O}}=\left\{f \in C^{\infty}\left(\mathbb{R}^{n}, \mathcal{M}\left(k, \mathcal{K}_{1}, \eta\right)\right): \forall(\hat{x}, \varepsilon, \xi, \omega) \in \mathcal{K} \times E_{m-1},\right. \\
& \left.H_{\{0, \ldots, k\}}^{m-1}(f(\hat{x}), \varepsilon, \xi, \omega) \neq 0\right\}
\end{aligned}
$$

is an open dense subset of $C^{\infty}\left(\mathbb{R}^{n}, \mathcal{M}\left(k, \mathcal{K}_{1}, \eta\right)\right)$. Then $\mathscr{O}_{m-1}:=\{\tau \circ f: f \in \tilde{\mathscr{O}}\}$ where $\tau$ is the target map is an open dense subset of $\mathscr{N}\left(k, \mathcal{K}_{1}, \eta\right)$ and

$$
\begin{array}{r}
\mathscr{O}_{m-1}=\left\{\delta \in \mathscr{N}\left(k, \mathcal{K}_{1}, \eta\right): \forall\left(\hat{x}_{0}, \varepsilon_{0}, \xi_{0}, \omega_{0}\right) \in \mathcal{K} \times E_{m-1},\right. \\
\left.\quad H_{\{0, \ldots, k\}}^{m-1}\left(j^{k} \delta\left(\hat{x}_{0}\right), \varepsilon_{0}, \xi_{0}, \omega_{0}\right) \neq 0\right\} .
\end{array}
$$

It concludes the induction and the proof. 
Proof of Theorem 2.4. Applying Proposition 3.5 to $m=0$ and recalling the definition of $H_{\{0, \ldots, k\}}^{0}$, we immediately get the main Theorem 2.4 .

A straightforward consequence of Theorem 2.4 is the following corollary, that deals with the observability of (11), as announced in Remark 2.5.

Corollary 3.6. Assume that $(C, A)$ and $(C, B)$ are observable pairs. Assume that $0 \notin \mathcal{K}_{1}$. Then there exist $\eta>0, k \in \mathbb{N}$ and an open dense subset $\mathscr{O} \subset \mathscr{N}\left(k, \mathcal{K}_{1}, \eta\right)$ such that for all $\left(\delta, \hat{x}_{0}, \varepsilon_{0}, \xi_{0}\right) \in \mathscr{O} \times \mathcal{K}$, system (11) is observable in any time $T>0$ for the control $u=(\lambda+\delta) \circ \hat{x}$, where $\hat{x}$ follows (4) with initial conditions $\left(\hat{x}_{0}, \varepsilon_{0}, \xi_{0}\right)$ and feedback perturbation $\delta$.

Proof. Applying Proposition 3.5 to $m=0$, we find that there exist $\eta>0, k \in \mathbb{N}$ and an open dense subset $\mathscr{O} \subset \mathscr{N}\left(k, \mathcal{K}_{1}, \eta\right)$ such that for all $\left(\delta, \hat{x}_{0}, \varepsilon_{0}, \xi_{0}, \omega_{0}\right) \in \mathscr{O} \times \mathcal{K} \times E_{0}$, $H_{\{0, \ldots, k\}}^{0}\left(j^{k} \delta\left(\hat{x}_{0}\right), \varepsilon_{0}, \xi_{0}, \omega_{0}\right) \neq 0$. Let $\left(\delta, \hat{x}_{0}, \varepsilon_{0}, \xi_{0}, \omega_{0}\right) \in \mathscr{O} \times \mathcal{K} \times \mathbb{S}^{n-1}$, and let $(\hat{x}, \varepsilon, \xi, \omega)$ denote the solution of (44) with initial conditions $\left(\hat{x}_{0}, \varepsilon_{0}, \xi_{0}, \omega_{0}\right)$. From the definition of $H_{\{0, \ldots, k\}}^{0}$ it follows that there exists $i \in \mathbb{N}$ such that $C \omega^{(i)}(0) \neq 0$. Consequently, $\left.C \omega\right|_{[0, T]} \not \equiv 0$, which was to be proved.

As stated in Remark [2.5, we now want to complete the compact $\mathcal{K}_{1}$ with a neighborhood of zero as in Corollary 2.6. We do so in the following section.

\subsection{Observability near the target and proof of Corollary 2.6}

We use Theorem 2.4 to prove Corollary 2.6. In order to do so, we need the following notations and lemmas. For any control $u \in C^{\infty}\left(\mathbb{R}_{+}, \mathbb{R}\right)$, let $\Phi_{u}: \mathbb{R}_{+} \rightarrow \operatorname{End}\left(\mathbb{R}^{n}\right)$ be the flow of the time-varying linear ordinary differential equation (77). So $\Phi_{u}(t) \omega_{0}$ is the solution of (7) at time $t \in \mathbb{R}_{+}$with initial condition $\omega_{0} \in \mathbb{R}^{n}$. Notice for instance that $\Phi_{0}(t)=\mathrm{e}^{A t}$. Recall that an input $u \in C^{\infty}\left(\mathbb{R}_{+}, \mathbb{R}\right)$ is said to make system (1) observable in time $T>0$ if for all $\omega_{0} \in \mathbb{S}^{n-1}$ there exists $t \in[0, T]$ such that $C \Phi_{u}(t) \omega_{0} \neq 0$.

Lemma 3.7. Let $T>0, \eta_{0}=\max \left\{\left|C \Phi_{0}(t) \omega_{0}\right|: t \in[0, T], \omega_{0} \in \mathbb{S}^{n-1}\right\}$ and $u \in C^{\infty}\left(\mathbb{R}_{+}, \mathbb{R}\right)$. If

$$
\forall t \in[0, T], \forall \omega_{0} \in \mathbb{S}^{n-1}, \quad\left|C \Phi_{u}(t) \omega_{0}-C \Phi_{0}(t) \omega_{0}\right|<\eta_{0},
$$

then u makes system (11) observable in time $T$.

Proof. Let $t \in[0, T]$ and $\omega_{0} \in \mathbb{S}^{n-1}$ be such that $\left|C \Phi_{0}(t) \omega_{0}\right|=\eta_{0}$. Using (16), we get

$$
\left|C \Phi_{u}(t) \omega_{0}\right| \geqslant\left|C \Phi_{0}(t) \omega_{0}\right|-\left|C \Phi_{u}(t) \omega_{0}-C \Phi_{0}(t) \omega_{0}\right|>0
$$

which shows that $u$ makes system (1) observable in time $T$.

Lemma 3.8. Let $T>0$. Let $M=\sup \left\{\left\|\Phi_{0}(t)\right\|: t \in[0, T]\right\}$. Let $u \in C^{\infty}\left(\mathbb{R}_{+}, \mathbb{R}\right)$ and let $u_{M}=\sup \{|u(t)|: t \in[0, T]\}$. Then there exists a constant $K>0$ such that for all $t \in[0, T]$ and all $\omega_{0} \in \mathbb{S}^{n-1}$,

$$
\left|\Phi_{u}(t) \omega_{0}-\Phi_{0}(t) \omega_{0}\right|<M K u_{M} \mathrm{e}^{K u_{M}} .
$$

Proof. By the variation of constants formula, for all $t \in[0, T]$ and all $\omega_{0} \in \mathbb{S}^{n-1}$,

$$
\Phi_{u}(t) \omega_{0}-\Phi_{0}(t) \omega_{0}=\int_{0}^{t} \Phi_{0}(t-s) B u(s) \Phi_{u}(s) \mathrm{d} s \omega_{0}
$$


Iterating integrals, we get a (formal) series expansion

$$
\int_{0}^{s_{0}} \Phi_{0}\left(s_{0}-s_{1}\right) B u(s) \Phi_{u}(s) \mathrm{d} s_{1}=\sum_{k=0}^{+\infty} J_{k}
$$

where

$$
J_{k}=\int_{0}^{s_{0}} \cdots \int_{0}^{s_{k}} \Psi_{k}\left(s_{0}, \ldots, s_{k+1}\right) \Phi_{0}\left(s_{k+1}\right) u\left(s_{0}\right) \cdots u\left(s_{k+1}\right) \mathrm{d} s_{1} \cdots \mathrm{d} s_{k+1}
$$

with $\Psi_{k}\left(s_{0}, \ldots, s_{k+1}\right)=\Phi_{0}\left(s_{0}-s_{1}\right) B \cdots \Phi_{0}\left(s_{k}-s_{k+1}\right) B$.

Then $\left\|\Psi_{k}\left(s_{0}, \ldots, s_{k+1}\right)\right\| \leqslant M^{k+1}\|B\|^{k+1}$ and

$$
\left\|J_{k}\right\| \leqslant M^{k+2}\|B\|^{k+1} u_{M}^{k+1} \int_{0}^{s_{0}} \cdots \int_{0}^{s_{k}} \mathrm{~d} s_{1} \cdots \mathrm{d} s_{k+1} \leqslant M^{k+2}\|B\|^{k+1} u_{M}^{k+1} \frac{T^{k+1}}{(k+1) !} .
$$

Thus

$$
\begin{aligned}
\sum_{k=0}^{+\infty}\left\|J_{k}\right\| & \leqslant \sum_{k=0}^{+\infty} M^{k+2}\|B\|^{k+1} u_{M}^{k+1} \frac{T^{k+1}}{(k+1) !} \\
& \leqslant M^{2}\|B\| u_{M} T \sum_{k=0}^{+\infty} M^{k}\|B\|^{k} u_{M}^{k} \frac{T^{k}}{k !}
\end{aligned}
$$

which proves the convergence of the series expansion (18) and inequality (17) with $K=$ $M\|B\| T$.

Proposition 3.9. Assume that the pair $(C, A)$ is observable. Assume that 0 is in the interior of $\mathcal{K}_{1}$. Let $T>0$. Then there exists $R>0$ such that $B(0, R) \subset \mathcal{K}_{1}$ and $\eta_{1}>0$ such that the following property holds:

Let $(\hat{x}, \varepsilon, \xi, \omega)$ be the solution of (44) with initial condition $\left(\hat{x}_{0}, \varepsilon_{0}, \xi_{0}, \omega_{0}\right) \in B(0, R) \times \mathbb{R}^{n} \times$ $\mathcal{S} \times \mathbb{S}^{n-1}$. Let $\delta \in C^{\infty}\left(\mathbb{R}^{n}, \mathbb{R}\right)$ such that $\delta(0)=0$ and $\sup \left\{|\delta(x)|: x \in \mathcal{K}_{1}\right\}<\eta_{1}$. If $\hat{x}(t) \in B(0, R)$ for all $t \in[0, T]$, then the control $u: t \mapsto(\lambda+\delta)(\hat{x}(t))$ makes system (1) observable in time $T$.

Proof. Let $T>0$ and $\eta_{0}$ be as in the statement of Lemma 3.7. The observability of the pair $(C, A)$ yields $\eta_{0}>0$. Let $\eta_{1}>0$ be such that $M K \eta_{1} \mathrm{e}^{K \eta_{1}}<\eta_{0}$. For all $R>0$ and all $\delta \in C^{\infty}\left(\mathbb{R}^{n}, \mathbb{R}\right)$ satisfying $\delta(0)=0$ and $\sup \left\{|\delta(x)|: x \in \mathcal{K}_{1}\right\}<\eta_{1}$, let $u_{M}(R, \delta)=\sup \{\mid(\lambda+$ $\delta)(x) \mid: x \in B(0, R)\}$. Since $\lambda+\delta$ is continuous and $\lambda(0)=\delta(0)=0, u_{M}(\cdot, \delta)$ is a continuous non decreasing function on $\mathbb{R}_{+}$such that $u_{M}(0,0)=0$ and $u_{M}(R, \delta) \leqslant u_{M}(R, 0)+\eta_{1}$. Then, we can choose $R>0$ such that $M K\left(u_{M}(R, 0)+\eta_{1}\right) \mathrm{e}^{K\left(u_{M}(R, 0)+\eta_{1}\right)}<\eta_{0}$. Since $u_{M}(\cdot, 0)$ is non decreasing, it is possible to choose $R$ such that $B(0, R) \subset \mathcal{K}_{1}$.

Now, fix $\delta \in C^{\infty}\left(\mathbb{R}^{n}, \mathbb{R}\right)$ satisfying $\delta(0)=0$ and $\sup \left\{|\delta(x)|: x \in \mathcal{K}_{1}\right\}<\eta_{1}$. Let $(\hat{x}, \varepsilon, \xi, \omega)$ be the solution of (4) with initial condition $\left(\hat{x}_{0}, \varepsilon_{0}, \xi_{0}, \omega_{0}\right) \in B(0, R) \times \mathbb{R}^{n} \times \mathcal{S} \times \mathbb{S}^{n-1}$. Then $M K u_{M}(R, \delta) \mathrm{e}^{K u_{M}(R, \delta)}<\eta_{0}$. Hence, from Lemmas 3.7 and [3.8, if $\hat{x}(t) \in B(0, R)$ for all $t \in[0, T]$, then the control $u: t \mapsto(\lambda+\delta)(\hat{x}(t))$ makes system (10) observable in time $T$.

Proof of Corollary 2.6. Let $R>0$ and $\eta_{1}$ be as in Proposition 3.9. Let $r \in(0, R)$ and $\rho \in(0, r)$. We apply Corollary 3.6 to the compact $\mathcal{K}_{1} \backslash B(0, r)$. Since the statement holds for some $\eta$ small enough, we assume without loss of generality that $\eta<\eta_{1}$ : there exist $\eta \in\left(0, \eta_{1}\right)$, $k \in \mathbb{N}$ and an open dense subset $\mathscr{O} \subset \mathscr{N}\left(k, \mathcal{K}_{1} \backslash B(0, r), \eta\right)$ such that for all $\left(\delta, \hat{x}_{0}, \varepsilon_{0}, \xi_{0}\right) \in$ $\mathscr{O} \times\left(\mathcal{K}_{1} \backslash B(0, r)\right) \times \mathcal{K}_{2} \times \mathcal{K}_{3}$, system (11) is observable in any time $T>0$ for the control $u=(\lambda+\delta) \circ \hat{x}$, where $\hat{x}$ follows (4) with initial conditions $\left(\hat{x}_{0}, \varepsilon_{0}, \xi_{0}\right)$ and feedback perturbation $\delta$. 
Let

$$
\mathscr{O}^{\prime}=\left\{\tilde{\delta} \in \mathscr{N}\left(k, \mathcal{K}_{1}, \eta\right) \cap \mathcal{V}_{\rho}: \exists \delta \in \mathscr{O}, \forall x \in \mathcal{K}_{1} \backslash B(0, r), \tilde{\delta}(x)=\delta(x)\right\} .
$$

Then $\mathscr{O}^{\prime}$ is open and dense in $\mathscr{N}\left(k, \mathcal{K}_{1}, \eta\right) \cap \mathcal{V}_{\rho}$ (in the Whitney $C^{\infty}$ induced topology) since $\mathscr{O}$ is open and dense in $\mathscr{N}\left(k, \mathcal{K}_{1} \backslash B(0, r), \eta\right)$. Moreover, if $\tilde{\delta} \in \mathscr{O}^{\prime}$, then system (1D) is still observable in any time $T>0$ for the control $u=(\lambda+\tilde{\delta}) \circ \hat{x}$ with initial conditions $\left(\hat{x}_{0}, \varepsilon_{0}, \xi_{0}\right)$ in $\left(\mathcal{K}_{1} \backslash B(0, r)\right) \times \mathcal{K}_{2} \times \mathcal{K}_{3}$.

Let $\left(\tilde{\delta}, \hat{x}_{0}, \varepsilon_{0}, \xi_{0}\right) \in \mathscr{O}^{\prime} \times \mathcal{K}$. If $\hat{x}_{0} \notin B(0, r)$, then the result holds from above. On the other hand, assume that $\hat{x}_{0} \in B(0, r)$. If $\hat{x}(t) \in B(0, R)$ for all $t \in[0, T]$, then according to Proposition 3.9, (11) is observable in time $T$ for the control $u=(\lambda+\tilde{\delta}) \circ \hat{x}$. Otherwise, there exists $t_{0} \in(0, T)$ such that $\hat{x}\left(t_{0}\right) \notin B(0, r)$. Apply Corollary 3.6 with the new initial condition $\left(\hat{x}\left(t_{0}\right), \varepsilon\left(t_{0}\right), \xi\left(t_{0}\right)\right)$ and with the same perturbation $\tilde{\delta}$. Then (11) is observable in time $T>t_{0}$ for the control $u=(\lambda+\tilde{\delta}) \circ \hat{x}$.

Proof of Corollary 2.7. Let $T>0$ and $\lambda \in \Lambda$. Let $R, \eta, k$ and $\mathscr{O}$ be as in Corollary 2.6. Since $\mathscr{O}$ is dense (in the Whitney $C^{\infty}$ topology) in $\mathscr{N}\left(k, \mathcal{K}_{1}, \eta\right) \cap \mathcal{V}_{R}$, for all neighborhood $\mathscr{U}$ of $\lambda \in \Lambda$, there exists $\delta \in \mathscr{O}$ such that $\lambda+\delta \in \mathscr{U} \cap \Lambda_{T}$. Hence, $\Lambda_{T}$ is a dense subset of $\Lambda$. Moreover,

$$
\begin{aligned}
\Lambda_{T} & =\left\{\lambda \in \Lambda: \forall\left(\hat{x}_{0}, \varepsilon_{0}, \xi_{0}, \omega_{0}\right) \in \mathcal{K} \times \mathbb{S}^{n-1}, \exists t \in[0, T], C \omega(t) \neq 0\right\} \\
& =\bigcap_{\left(\hat{x}_{0}, \varepsilon_{0}, \xi_{0}, \omega_{0}\right) \in \mathcal{K} \times \mathbb{S}^{n-1}} h_{\hat{x}_{0}, \varepsilon_{0}, \xi_{0}, \omega_{0}}^{-1}\left(\mathcal{C}^{\infty}([0, T], \mathbb{R}) \backslash\{0\}\right)
\end{aligned}
$$

where $h_{\hat{x}_{0}, \varepsilon_{0}, \xi_{0}, \omega_{0}}: \Lambda \rightarrow C^{\infty}([0, T], \mathbb{R})$ is given by $h_{\hat{x}_{0}, \varepsilon_{0}, \xi_{0}, \omega_{0}}(\lambda)=\left.C \omega\right|_{[0, T]}$ where $\omega$ is the solution of (4) with initial condition $\left(\hat{x}_{0}, \varepsilon_{0}, \xi_{0}, \omega_{0}\right)$ and $\delta \equiv 0$. The map $h$ is continuous, the set $\mathcal{C}^{\infty}([0, T], \mathbb{R}) \backslash\{0\}$ is open and the set $\mathcal{K} \times \mathbb{S}^{n-1}$ is compact. Thus $\Lambda_{T}$ is open in $\Lambda$.

\section{Application to classical observers}

In this section, we show that there exist observers such that the key hypotheses (FC) and (NFOT) are satisfied. In particular, we show that both the Luenberger observer and the Kalman observer satisfy these hypotheses, as stated in Theorem 2.9. Hence, the main Theorem 2.4 and its Corollary 2.6 apply to these observers. While (FC) has already been studied for such observers (see e.g. [5, 10]), (NFOT) is more difficult to check, and relies on the fact that the observer dynamics is somehow compatible with the Kalman observability decomposition.

For the sake of generality, we state the results of this section for an arbitrary output dimension $m$ (i.e. $\left.C \in \mathcal{L}\left(\mathbb{R}^{n}, \mathbb{R}^{m}\right)\right)$. Let $\mathcal{S}_{n} \subset \operatorname{End}\left(\mathbb{R}^{n}\right)$ denote the subset of real positivedefinite symmetric endomorphism on $\mathbb{R}^{n}$.

Regarding hypothesis (FC), the following result is well-known.

Proposition 4.1. Assume that $\lambda$ is bounded over $D(\lambda)$. Let $Q \in \mathcal{S}_{n}$. For all $\xi \in \mathcal{S}_{n}$ and all $u \in \mathbb{R}$, consider the following well-known observers:

$$
\begin{array}{lr}
f^{\text {Luenberger }}(\xi, u)=0 & \text { (Luenberger observer) } \\
f_{Q}^{\text {Kalman }}(\xi, u)=\xi A_{u}^{*}+A_{u} \xi+Q-\xi C^{*} C \xi & \text { (Kalman observer) }
\end{array}
$$

and $L(\xi)=\xi C^{*}$. Then the coupled system (44) given by $(f, L)$ satisfies the hypothesis (FC) for any $f \in\left\{f^{\text {Luenberger }}, f_{Q}^{\text {Kalman }}\right\}$. 
Let us investigate hypothesis (NFOT), First, we state sufficient conditions for it to hold, and then show that they are satisfied by both the Kalman and Luenberger observers.

For all $A_{0} \in C^{\infty}\left(\mathbb{R}_{+}, \operatorname{End}\left(\mathbb{R}^{n}\right)\right)$ and for all $C_{0} \in \mathcal{L}\left(\mathbb{R}^{n}, \mathbb{R}^{m}\right)$, let $f\left(\cdot, A_{0}, C_{0}\right)$ be a forward complete time-varying vector field over $\mathcal{S}_{n}$. Let $L: \mathcal{S}_{n} \times \mathcal{L}\left(\mathbb{R}^{n}, \mathbb{R}^{m}\right) \rightarrow \mathcal{L}\left(\mathbb{R}^{m}, \mathbb{R}^{n}\right)$. For all $T \in \operatorname{GL}\left(\mathbb{R}^{n}\right)$, for all $(\bar{A}, \bar{C}) \in \operatorname{End}\left(\mathbb{R}^{n}\right) \times \mathcal{L}\left(\mathbb{R}^{n}, \mathbb{R}^{m}\right)$ and for all $\xi \in \mathcal{S}_{n}$, let $(\bar{f}, \bar{L})$ be defined by

$$
\left\{\begin{array}{l}
\bar{f}\left(T \xi T^{*}, T \bar{A} T^{-1}, \bar{C} T^{-1}\right)=T f(\xi, \bar{A}, \bar{C}) T^{*} \\
\bar{L}\left(T \xi T^{*}, \bar{C} T^{-1}\right)=T L(\xi, \bar{C}) .
\end{array}\right.
$$

For all $(\bar{A}, \bar{C}, \bar{b}) \in \operatorname{End}\left(\mathbb{R}^{n}\right) \times \mathcal{L}\left(\mathbb{R}^{n}, \mathbb{R}^{m}\right) \times \mathbb{R}^{n}$, we consider the following dynamical observer system

$$
\left\{\begin{array}{l}
\dot{\hat{x}}=\bar{A} \hat{x}+\bar{b}-\bar{L}(\xi, \bar{C}) \bar{C} \varepsilon \\
\dot{\varepsilon}=(\bar{A}-\bar{L}(\xi, \bar{C}) \bar{C}) \varepsilon \\
\dot{\xi}=\bar{f}(\xi, \bar{A}, \bar{C}) .
\end{array}\right.
$$

For all $k \in\{1, \ldots, n\}$, let $(\bar{A}, \bar{C}) \in \operatorname{End}\left(\mathbb{R}^{n}\right) \times \mathcal{L}\left(\mathbb{R}^{n}, \mathbb{R}^{m}\right)$ having the following structure:

$$
\bar{A}=\left(\begin{array}{cc}
A_{11} & 0 \\
A_{21} & A_{22}
\end{array}\right), \quad \bar{C}=\left(\begin{array}{ll}
C_{1} & 0
\end{array}\right),
$$

with suitable matrices $A_{11} \in \operatorname{End}\left(\mathbb{R}^{k}\right), A_{21} \in \mathcal{L}\left(\mathbb{R}^{k}, \mathbb{R}^{n-k}\right), A_{22} \in \operatorname{End}\left(\mathbb{R}^{n-k}\right)$ and $C_{1} \in$ $\mathcal{L}\left(\mathbb{R}^{k}, \mathbb{R}^{m}\right)$. For any solution of (20), set similarly

$$
\hat{x}=\left(\begin{array}{l}
\hat{x}_{1} \\
\hat{x}_{2}
\end{array}\right), \quad \varepsilon=\left(\begin{array}{l}
\varepsilon_{1} \\
\varepsilon_{2}
\end{array}\right), \quad \bar{b}=\left(\begin{array}{l}
b_{1} \\
b_{2}
\end{array}\right), \quad \xi=\left(\begin{array}{ll}
\xi_{11} & \xi_{12} \\
\xi_{12}^{*} & \xi_{22}
\end{array}\right) .
$$

Proposition 4.2. Assume that the pair $(C, A)$ is observable. Assume that for all $T \in \mathrm{GL}\left(\mathbb{R}^{n}\right)$, for all $(\bar{f}, \bar{L})$ as in (19), for all $k \in\{1, \ldots, n\}$ and for all $(\bar{A}, \bar{C}) \in \operatorname{End}\left(\mathbb{R}^{n}\right) \times \mathcal{L}\left(\mathbb{R}^{n}, \mathbb{R}^{m}\right)$ as in (21), the following hypotheses hold.

H1. There exists $\left(f_{11}, L_{1}\right)$ such that

$$
\left\{\begin{array}{l}
\dot{\hat{x}}_{1}=A_{11} \hat{x}_{1}+b_{1}-L_{1}\left(\xi_{11}, C_{1}\right) C_{1} \varepsilon_{1} \\
\dot{\varepsilon}_{1}=\left(A_{11}-L_{1}\left(\xi_{11}, C_{1}\right) C_{1}\right) \varepsilon_{1} \\
\dot{\xi}_{11}=f_{11}\left(\xi_{11}, A_{11}, C_{1}\right)
\end{array}\right.
$$

where $\left(f_{11}, L_{1}\right)$ is such that

$$
\bar{f}(\xi, \bar{A}, \bar{C})=\left(\begin{array}{cc}
f_{11}\left(\xi_{11}, A_{11}, C_{1}\right) & * \\
* & *
\end{array}\right), \quad \bar{L}(\xi, \bar{C})=\left(\begin{array}{c}
L_{1}\left(\xi_{11}, C_{1}\right) \\
*
\end{array}\right) .
$$

H2. If $\left(C_{1}, A_{11}\right) \in \mathcal{L}\left(\mathbb{R}^{k}, \mathbb{R}^{m}\right) \times \operatorname{End}\left(\mathbb{R}^{k}\right)$ is an observable pair, then the solutions of (20) are such that for any initial conditions, $L_{11}\left(\xi_{11}(t), C_{1}\right) C_{1} \varepsilon_{1}(t) \rightarrow 0$ as $t \rightarrow+\infty$.

H3. For all $\xi_{11} \in \mathcal{S}_{k}$ and all $C_{1} \in \mathcal{L}\left(\mathbb{R}^{k}, \mathbb{R}^{m}\right)$, $\operatorname{ker} L_{1}\left(\xi_{11}, C_{1}\right) \cap \operatorname{Im} C_{1}=\{0\}$.

Then the coupled system (4) given by $\left(f\left(\cdot, A_{u}, C\right), L(\cdot, C)\right)$ satisfies the hypothesis (NFOT). 
Remark 4.3. In the case where $T$ is the identity matrix and $k=n,(\mathrm{H} 1)$ is clearly satisfied, (H2) means that the correction term $L(\xi, \bar{C}) \bar{C} \varepsilon$ converges to zero for any observable pair $(A, C)$, and (H3) means that the correction term is null if and only if $\bar{C} \varepsilon=0$. We will see in Theorem 2.9 that these hypotheses are clearly satisfied for the Luenberger and Kalman observers.

Remark 4.4. Hypothesis (H1) can be seen as a compatibility condition between the observer dynamics and the Kalman observability decomposition: when $\bar{A}$ is of the standard form (21), the observer acts autonomously on the upper left matrix block, which will correspond to the observable part of the system.

This proposition is a consequence of the series of lemmas that follows. Until the end of the proof of Proposition 4.2, assume that its hypotheses are satisfied. For any $\mu: \mathbb{R}^{n} \rightarrow \mathbb{R}, F_{\mu}$ denotes the vector field over $\mathbb{R}^{n}$ given by $F_{\mu}(x)=A_{\mu(x)} x+b \mu(x)$.

Lemma 4.5. For all $R>0$, there exists $\eta>0$ such that for all $\delta \in \mathcal{V}_{R}$ satisfying $\sup \{|\delta(x)|$ : $\left.x \in \mathcal{K}_{1}\right\}<\eta, 0$ is the unique equilibrium point of $F_{\lambda+\delta}$ lying in $\mathcal{K}_{1}$.

Proof. Let $R>0$ and $\delta \in \mathcal{V}_{R}$. Let $x \in \mathcal{K}_{1}$ be such that $F_{\lambda+\delta}(x)=0$. Then,

$$
0=F_{\lambda+\delta}(x)=F_{\lambda}(x)+\delta(x)(B x+b) .
$$

Then $\left|F_{\lambda}(x)\right|=|\delta(x)||B x+b|$. Set $C_{1}=\inf \left\{\left|F_{\lambda}(x)\right|: x \in \mathcal{K}_{1} \backslash B(0, R)\right\}$. Since 0 is not in the closure of $\mathcal{K}_{1} \backslash B(0, R)$, we get by uniqueness of the equilibrium point of $F_{\lambda}$ that $C_{1}>0$. Set also $C_{2}=\sup \left\{|B x+b|: x \in \mathcal{K}_{1}\right\}$. Since $\mathcal{K}_{1}$ is compact, $C_{2}<+\infty$. Set $\eta=\frac{C_{1}}{C_{2}}$. Assume that $\sup \left\{|\delta(x)|: x \in \mathcal{K}_{1}\right\}<\eta$. Then,

$$
F_{\lambda}(x) \leqslant \eta|B x+b| \leqslant C_{1} .
$$

Hence $x \in B(0, R)$ by definition of $C_{1}$. Then $\delta(x)=0$. Hence $F_{\lambda}(x)=0$. Thus, $x=0$ since 0 is the unique equilibrium point of $F_{\lambda}$. Moreover, by definition of $\mathcal{V}_{R}, F_{\lambda+\delta}(0)=0$.

Lemma 4.6. Assume that the pair $(C, A)$ is observable. Let $\left(u_{0}, \hat{x}_{0}, \varepsilon_{0}, \xi_{0}\right) \in \mathbb{R} \times \mathbb{R}^{n} \times \mathbb{R}^{n} \times \mathcal{S}$. Let $(\hat{x}, \varepsilon, \xi)$ be the solution of (3) given by the initial condition $\left(\hat{x}_{0}, \varepsilon_{0}, \xi_{0}\right)$ and the constant input $u \equiv u_{0}$. If $\hat{x}$ is constant, then for all $t \in \mathbb{R}_{+}, L(\xi(t), C) C \varepsilon(t)=0$.

Proof. Let $\left(u_{0}, \hat{x}_{0}, \varepsilon_{0}, \xi_{0}\right) \in \mathbb{R} \times \mathbb{R}^{n} \times \mathbb{R}^{n} \times \mathcal{S}$. Let $(\hat{x}, \varepsilon, \xi)$ be the solution of (3) given by the initial condition $\left(\hat{x}_{0}, \varepsilon_{0}, \xi_{0}\right)$ and the constant input $u \equiv u_{0}$. Assume that $\hat{x}$ is constant, i.e. $\hat{x} \equiv \hat{x}_{0}$. Set $A_{0}=A+u_{0} B$ and $b_{0}=b u_{0}$. Then $\dot{\hat{x}} \equiv 0$ yields

$$
A_{0} \hat{x}+b_{0}-L(\xi, C) C \varepsilon \equiv 0 .
$$

Since $\hat{x}$ is constant, so is $L(\xi) C \varepsilon$. Then, set $K=L(\xi, C) C \varepsilon$. It remains to show that $K=0$.

Let $k=\operatorname{rank} \mathcal{O}\left(C, A_{0}\right)$ where $\mathcal{O}\left(C, A_{0}\right)$ is defined by (2) Since $C \neq 0$ (since $\left(C, A_{0}\right)$ is observable), $k \geqslant 1$. According to the Kalman observability decomposition, there exists an invertible endomorphism $T \in \mathrm{GL}\left(\mathbb{R}^{n}\right)$ such that $\bar{A}=T A_{0} T^{-1}$ and $\bar{C}=C T^{-1}$ have the following structure:

$$
\bar{A}=\left(\begin{array}{cc}
A_{11} & 0 \\
A_{21} & A_{22}
\end{array}\right), \quad \bar{C}=\left(\begin{array}{ll}
C_{1} & 0
\end{array}\right),
$$

with suitable matrices $A_{11} \in \operatorname{End}\left(\mathbb{R}^{k}\right), A_{21} \in \mathcal{L}\left(\mathbb{R}^{k}, \mathbb{R}^{n-k}\right), A_{22} \in \operatorname{End}\left(\mathbb{R}^{n-k}\right)$ and $C_{1} \in$ $\mathcal{L}\left(\mathbb{R}^{k}, \mathbb{R}^{m}\right)$. Moreover, the pair $\left(C_{1}, A_{11}\right)$ is observable. For the sake of readability, we omit 
the horizontal bars over the submatrices (for instance, $A_{11}$ is a submatrix of $\bar{A}$ and not of $A$ ). Similarly, set

$$
\begin{aligned}
& \bar{x}=T x=\left(\begin{array}{l}
x_{1} \\
x_{2}
\end{array}\right), \quad \overline{\hat{x}}=T \hat{x}=\left(\begin{array}{l}
\hat{x}_{1} \\
\hat{x}_{2}
\end{array}\right), \quad \bar{\varepsilon}=T \varepsilon=\left(\begin{array}{l}
\varepsilon_{1} \\
\varepsilon_{2}
\end{array}\right), \\
& \bar{b}_{0}=T b_{0}=\left(\begin{array}{l}
b_{1} \\
b_{2}
\end{array}\right), \quad \bar{K}=T K=\left(\begin{array}{l}
K_{1} \\
K_{2}
\end{array}\right), \quad \bar{\xi}=T \xi T^{*}=\left(\begin{array}{ll}
\xi_{11} & \xi_{12} \\
\xi_{12}^{*} & \xi_{22}
\end{array}\right) .
\end{aligned}
$$

Then, according to (19), we have the following observed control system on $\bar{x}$, and the corresponding observer:

$$
\left\{\begin{array}{l}
\dot{\bar{x}}=\bar{A} \bar{x}+\bar{b}_{0} \\
y=\bar{C} \bar{x} \\
\dot{\overline{\hat{x}}}=\bar{A} \overline{\hat{x}}+\bar{b}_{0}-\bar{L}(\xi, \bar{C}) \bar{C} \bar{\varepsilon} \\
\dot{\bar{\varepsilon}}=(\bar{A}-\bar{L}(\xi, \bar{C}) \bar{C}) \bar{\varepsilon} \\
\dot{\bar{\xi}}=\bar{f}(\bar{\xi}, \bar{A}, \bar{C}) .
\end{array}\right.
$$

Then, according to hypothesis (H1), we can write

$$
\left\{\begin{array}{l}
\dot{\xi}_{11}=f_{11}\left(\xi_{11}, A_{11}\right) \\
\dot{\hat{x}}_{1}=A_{11} \hat{x}_{1}+b_{1}-L_{1}\left(\xi_{11}, C_{1}\right) C_{1} \varepsilon_{1} \\
\dot{\varepsilon}_{1}=\left(A_{11}-L_{1}\left(\xi_{11}, C_{1}\right) C_{1}\right) \varepsilon_{1} .
\end{array}\right.
$$

Since the pair $\left(C_{1}, A_{11}\right)$ is observable, (H1) and (H2) yield $L_{1}\left(\xi_{11}(t), C_{1}\right) C_{1} \varepsilon_{1}(t) \rightarrow 0$ as $t \rightarrow$ $+\infty$. The equality $K_{1}=L_{1}\left(\xi_{11}(t), C_{1}\right) C_{1} \varepsilon_{1}(t)$ thus yields $K_{1}=0$. Then, by hypotheses (H1) and (H3), $\bar{C} \varepsilon \equiv C_{1} \varepsilon_{1} \equiv 0$. Hence $K=0$. Finally, we have $K=T^{-1} \bar{K}=0$.

Lemma 4.7. Let $\left(\delta, \hat{x}_{0}, \varepsilon_{0}, \xi_{0}\right) \in C^{\infty}\left(\mathbb{R}^{n}, \mathbb{R}\right) \times \mathcal{K}$. Let $(\hat{x}, \varepsilon, \xi)$ be the solution of (44) given by $\left(\delta, \hat{x}_{0}, \varepsilon_{0}, \xi_{0}\right)$. Set $u_{0}=(\lambda+\delta)\left(\hat{x}_{0}\right)$. Let $\left(\hat{x}_{\omega}, \varepsilon_{\omega}, \xi_{\omega}\right)$ be the solution of (B) given by the initial condition $\left(\hat{x}_{0}, \varepsilon_{0}, \xi_{0}\right)$ and the constant input $u \equiv u_{0}$. If $\hat{x}^{(i)}(0)=0$ for all $i \in \mathbb{N} \backslash\{0\}$, then $\hat{x}_{\omega}$ is constant and

$$
\left(\varepsilon_{\omega}^{(k)}(0), \xi_{\omega}^{(k)}(0)\right)=\left(\varepsilon^{(k)}(0), \xi^{(k)}(0)\right)
$$

for all $k \in \mathbb{N}$.

Proof. Assume that $\hat{x}^{(i)}(0)=0$ for all $i \in \mathbb{N} \backslash\{0\}$. Then, for all $i \in \mathbb{N} \backslash\{0\}$,

$$
A_{(\lambda+\delta)(\hat{x})}^{(i)}(0)=0
$$

According to the ODE version of the Cauchy-Kovalevskaya theorem, $\left(\hat{x}_{\omega}, \varepsilon_{\omega}, \xi_{\omega}\right)$ is analytic in a neighborhood of 0 . Hence, it is sufficient to show that

$$
\left(\hat{x}_{\omega}^{(k)}(0), \varepsilon_{\omega}^{(k)}(0), \xi_{\omega}^{(k)}(0)\right)=\left(\hat{x}^{(k)}(0), \varepsilon^{(k)}(0), \xi^{(k)}(0)\right)
$$

for all $k \in \mathbb{N}$. By definition of $(\hat{x}, \varepsilon, \xi)$ and $\left(\hat{x}_{\omega}, \varepsilon_{\omega}, \xi_{\omega}\right)$, we have

$$
\left(\hat{x}_{\omega}(0), \varepsilon_{\omega}(0), \xi_{\omega}(0)\right)=\left(\hat{x}_{0}, \varepsilon_{0}, \xi_{0}\right)=(\hat{x}(0), \varepsilon(0), \xi(0)) .
$$


Let $k \in \mathbb{N}$. Assume that for all $i \in\{0, \ldots, k\}$, (28) is satisfied. Then we prove that (28) is also satisfied for $i=k+1$. Using Faà di Bruno's formula and (27), we get

$$
\begin{aligned}
\xi^{(k+1)}(0) & =f\left(\xi, A_{(\lambda+\delta)(\hat{x})}, C\right)^{(k)}(0) \\
& =f\left(\xi, A_{(\lambda+\delta)(\hat{x}(0))}, C\right)^{(k)}(0) \\
& =f\left(\xi_{\omega}, A_{(\lambda+\delta)(\hat{x}(0))}, C\right)^{(k)}(0) \\
& =\xi_{\omega}^{(k+1)}(0) .
\end{aligned}
$$

(by induction hypothesis)

Likewise, we obtain $\varepsilon^{(k+1)}(0)=\varepsilon_{\omega}^{(k+1)}(0)$ and $\hat{x}^{(k+1)}(0)=\hat{x}_{\omega}^{(k+1)}(0)$.

Lemma 4.8. Assume that the pair $(C, A)$ is observable. Let $\left(\hat{x}_{0}, \varepsilon_{0}, \xi_{0}\right) \in \mathcal{K}$. Let $R>0, \eta>0$ as in Lemma 4.5 and $\delta \in \mathcal{V}_{R}$ satisfying $\sup \left\{|\delta(x)|: x \in \mathcal{K}_{1}\right\}<\eta$. Let $(\hat{x}, \varepsilon, \xi)$ be the solution of (44) given by $\left(\delta, \hat{x}_{0}, \varepsilon_{0}, \xi_{0}\right)$. If for all $i \in \mathbb{N} \backslash\{0\}, \hat{x}^{(i)}(0)=0$, then $\hat{x} \equiv \varepsilon \equiv 0$.

Proof. Assume that for all $i \in \mathbb{N} \backslash\{0\}, \hat{x}^{(i)}(0)=0$. Set $u_{0}=(\lambda+\delta)\left(\hat{x}_{0}\right)$. Let $\left(\hat{x}_{\omega}, \varepsilon_{\omega}, \xi_{\omega}\right)$ be the solution of (4) given by the initial condition $\left(\hat{x}_{0}, \varepsilon_{0}, \xi_{0}\right)$ and the constant input $u \equiv u_{0}$. According to Lemma 4.7, $\hat{x}_{\omega} \equiv \hat{x}_{0}$ and for all $k \in \mathbb{N},\left(\varepsilon_{\omega}^{(k)}(0), \xi_{\omega}^{(k)}(0)\right)=\left(\varepsilon^{(k)}(0), \xi^{(k)}(0)\right)$. Then, by Lemma 4.6, we get that $L\left(\xi_{\omega}, C\right) C \varepsilon_{\omega} \equiv 0$. Hence, $A_{u_{0}} \hat{x}_{\omega}+b u_{0} \equiv 0$ i.e. $A_{(\lambda+\delta)\left(\hat{x}_{0}\right)} \hat{x}_{\omega}(t)+$ $b(\lambda+\delta)\left(\hat{x}_{0}\right)=0$ for all $t \in \mathbb{R}_{+}$. In particular, at $t=0$ we have that $F_{\lambda+\delta}\left(\hat{x}_{0}\right)=0$. Hence, from Lemma 4.5, $\hat{x}_{0}=0$. By uniqueness of the solution of (4) for a given initial condition, it remains to prove that $\varepsilon_{0}=0$ in order to get that $\hat{x} \equiv \varepsilon \equiv 0$. Since the pair $(C, A)$ is observable, it is sufficient to prove that $C A^{k} \varepsilon_{0}=0$ for all $k \in \mathbb{N}$. We proceed by induction. From Lemma 4.6, $L\left(\xi_{\omega}(0), C\right) C \varepsilon_{\omega}(0)=0$. Then, according to hypothesis (H3), $C \varepsilon_{0}=C \varepsilon_{\omega}(0)=0$. Let $k \in \mathbb{N}$. Assume that $C A^{i} \varepsilon_{0}=0$ for all $i \in\{0, \ldots, k-1\}$. We prove in the following that $C A^{k} \varepsilon_{0}=0$. From Lemma 4.6. $\left(L\left(\xi_{\omega}, C\right) C \varepsilon_{\omega}\right)^{(i)}(0)=0$ for all $i \in \mathbb{N}$. Hence, by Lemma 4.7, we get for all $i \in \mathbb{N},(L(\xi, C) C \varepsilon)^{(i)}(0)=\left(L\left(\xi_{\omega}, C\right) C \varepsilon_{\omega}\right)^{(i)}(0)=0$ and then $C \varepsilon^{(i)}(0)=C A_{u_{0}}^{i} \varepsilon_{0}=C A^{i} \varepsilon_{0}$ since $u_{0}=(\lambda+\delta)\left(\hat{x}_{0}\right)=(\lambda+\delta)(0)=0$. Then,

$$
\begin{aligned}
0 & =\left(L\left(\xi_{\omega}, C\right) C \varepsilon_{\omega}\right)^{(k)}(0) \\
& =(L(\xi, C) C \varepsilon)^{(k)}(0) \\
& =\sum_{i=0}^{k}\left(\begin{array}{c}
k \\
i
\end{array}\right) L(\xi, C)^{(k-i)}(0) C \varepsilon^{(i)}(0) \\
& =\sum_{i=0}^{k}\left(\begin{array}{c}
k \\
i
\end{array}\right) L(\xi, C)^{(k-i)}(0) C A^{i} \varepsilon_{0} \\
& =L\left(\xi_{0}, C\right) C A^{k} \varepsilon_{0} .
\end{aligned}
$$

(by Lemma 4.6)

(by Lemma 4.7)

(by Leibniz rule)

(by induction hypothesis)

Thus, by hypothesis (H3), $C A^{k} \varepsilon_{0}=0$, which concludes the induction and the proof.

This concludes the series of lemmas necessary to prove Proposition 4.2 and Theorem 2.9. Proof of Proposition 4.2. The statement follows directly from the contrapositive of Lemma 4.8

Proof of Theorem 2.9. Recall that, according to Proposition 4.1, the Luenberger observer and the Kalman observer satisfy (FC), It remains to show that the sufficient conditions stated in the Proposition 4.2 are satisfied by these observers to conclude the proof of Theorem 2.9. 
Let $Q \in \mathcal{S}_{n}$. For all $(\bar{A}, \bar{C}) \in \operatorname{End}\left(\mathbb{R}^{n}\right) \times \mathcal{L}\left(\mathbb{R}^{n}, \mathbb{R}^{m}\right)$ and all $\xi \in \mathcal{S}_{n}$, let

$$
\begin{aligned}
& f^{\text {Luenberger }}(\xi, \bar{A}, \bar{C})=0 \\
& f_{Q}^{\text {Kalman }}(\xi, \bar{A}, \bar{C})=\xi \bar{A}^{*}+\bar{A} \xi+Q-\xi \bar{C}^{*} \bar{C} \xi
\end{aligned}
$$

(Luenberger observer)

(Kalman observer)

and $L(\xi, C)=\xi \bar{C}^{*}$. Let $f \in\left\{f^{\text {Luenberger }}, f_{Q}^{\text {Kalman }}\right\}$. According to Proposition 4.1, the timevarying vector field $f$ is forward complete. For all $T \in \operatorname{GL}\left(\mathbb{R}^{n}\right)$, for all $(\bar{A}, \bar{C}) \in \operatorname{End}\left(\mathbb{R}^{n}\right) \times$ $\mathcal{L}\left(\mathbb{R}^{n}, \mathbb{R}^{m}\right)$ and for all $\xi \in \mathcal{S}_{n}$, let $(\bar{f}, \bar{L})$ be defined by

$$
\left\{\begin{array}{l}
\bar{f}\left(T \xi T^{*}, T \bar{A} T^{-1}, \bar{C} T^{-1}\right)=T f(\xi, \bar{A}, \bar{C}) T^{*} \\
\bar{L}\left(T \xi T^{*}, \bar{C} T^{-1}\right)=T L(\xi, \bar{C}) .
\end{array}\right.
$$

Then

$$
\bar{L}\left(T \xi T^{*}, \bar{C} T^{-1}\right)=T L(\xi, \bar{C})=T \xi \bar{C}^{*}=T \xi T^{*}\left(\bar{C} T^{-1}\right)^{*}=L\left(T \xi T^{*}, \bar{C} T^{-1}\right) .
$$

Hence $\bar{L}=L$. Moreover, if $f=f^{\text {Luenberger }}$, then $\bar{f}=f=0$. Otherwise, if $f=f_{Q}^{\text {Kalman }}$ and then

$$
\begin{aligned}
\bar{f}\left(T \xi T^{*}, T \bar{A} T^{-1}, \bar{C} T^{-1}\right)= & T f(\xi, \bar{A}, \bar{C}) T^{*} \\
= & T \xi \bar{A}^{*}+\bar{A} \xi+Q-\xi \bar{C}^{*} \bar{C} \xi T^{*} \\
= & T \xi T^{*}\left(T \bar{A} T^{-1}\right)^{*}+\left(T \bar{A} T^{-1}\right) T \xi T^{*} \\
& +T Q T^{*}-T \xi T^{*}\left(\bar{C} T^{-1}\right)^{*} \bar{C} T^{-1} T \xi T^{*} \\
= & f_{T Q T^{*}}^{\text {Kalman }}\left(T \xi T^{*}, T \bar{A} T^{-1}, \bar{C} T^{-1}\right),
\end{aligned}
$$

Hence it is sufficient to prove that, for all $(\bar{A}, \bar{C}) \in \operatorname{End}\left(\mathbb{R}^{n}\right) \times \mathcal{L}\left(\mathbb{R}^{n}, \mathbb{R}^{m}\right)$ satisfying (21),$(f, L)$ satisfies hypotheses (H1), (H2)] and (H3), Hypothesis (H1) requires some computations to check that if $(\bar{A}, \bar{C})$ is of the form (21), then (22) is satisfied with

$$
f_{11}\left(\xi_{11}, \bar{A}_{11}, \bar{C}_{1}\right)= \begin{cases}0 & \text { if } f=f^{\text {Luenberger }} \\ \xi_{11} \bar{A}_{11}^{*}+\bar{A}_{11} \xi_{11}+Q_{11}-\xi_{11} \bar{C}_{1}^{*} \bar{C}_{1} \xi_{11} & \text { if } f=f_{Q}^{\text {Kalman }}\end{cases}
$$

and $L_{1}\left(\xi_{11}, \bar{C}_{1}\right)=\xi_{11} \bar{C}_{1}^{*}$. Hence, for any $f \in\left\{f^{\text {Luenberger }}, f_{Q}^{\text {Kalman }}\right\}, f_{11}$ is an observer of the same form than $f$ acting on $\mathbb{R}^{k}$. Hypothesis (H2) follows from the fact that these well-known observers guaranty that the correction term $L_{1}\left(\xi_{11}, \bar{C}_{1}\right) \bar{C}_{1} \varepsilon_{1}$ goes to 0 as soon as the pair $\left(\bar{C}_{1}, \bar{A}_{11}\right)$ is observable (see e.g. [5, Chapter 1, Theorems 3 and 4]). Hypothesis (H3)] is clear: for all $\xi_{11} \in \mathcal{S}_{k}$ and all $\bar{C}_{1} \in \mathcal{L}\left(\mathbb{R}^{k}, \mathbb{R}^{m}\right)$, if $\varepsilon_{1} \in \mathbb{R}^{k}$ is such that $\xi_{11} \bar{C}_{1}^{*} \bar{C}_{1} \varepsilon_{1}=0$, then $C_{1} \varepsilon_{1}=0$ since $\xi_{11}$ is invertible. Thus the conclusion of Proposition 4.2 holds.

\section{Acknowledgments}

The authors would like to thank Vincent Andrieu and Daniele Astolfi for many fruitful discussions. They would also like to thank the anonymous reviewer, for suggesting the addition of Corollary 2.7 to the paper. 


\section{References}

[1] R. Abraham and J. Robbin. Transversal mappings and flows. An appendix by Al Kelley. W. A. Benjamin, Inc., New York-Amsterdam, 1967.

[2] V. Andrieu and L. Praly. A unifying point of view on output feedback designs for global asymptotic stabilization. Automatica J. IFAC, 45(8):1789-1798, 2009.

[3] A. N. Atassi and H. K. Khalil. A separation principle for the stabilization of a class of nonlinear systems. IEEE Trans. Automat. Control, 44(9):1672-1687, 1999.

[4] P. Bernard, L. Praly, V. Andrieu, and H. Hammouri. On the triangular canonical form for uniformly observable controlled systems. Automatica J. IFAC, 85:293-300, 2017.

[5] G. Besançon. Nonlinear Observers and Applications. Lecture Notes in Control and Information Sciences. Springer Berlin Heidelberg, 2007.

[6] J.-M. Coron. On the stabilization of controllable and observable systems by an output feedback law. Math. Control Signals Systems, 7(3):187-216, 1994.

[7] F. Esfandiari and H. K. Khalil. Output feedback stabilization of fully linearizable systems. Internat. J. Control, 56(5):1007-1037, 1992.

[8] M. Fliess and I. Kupka. A finiteness criterion for nonlinear input-output differential systems. Siam Journal on Control and Optimization, 21, 091983.

[9] J.-P. Gauthier and I. Kupka. A separation principle for bilinear systems with dissipative drift. IEEE Trans. Automat. Control, 37(12):1970-1974, 1992.

[10] J.-P. Gauthier and I. Kupka. Deterministic observation theory and applications. Cambridge University Press, Cambridge, 2001.

[11] M. Golubitsky and V. Guillemin. Stable Mappings and Their Singularities. Graduate texts in mathematics. Springer, 1974.

[12] M. Goresky and R. MacPherson. Stratified Morse theory, volume 14 of Ergebnisse der Mathematik und ihrer Grenzgebiete (3) [Results in Mathematics and Related Areas (3)]. Springer-Verlag, Berlin, 1988.

[13] H. K. Khalil and F. Esfandiari. Semiglobal stabilization of a class of nonlinear systems using output feedback. IEEE Transactions on Automatic Control, 38(9):1412-1415, Sep. 1993.

[14] M. Lagache, U. Serres, and J. Gauthier. Exact output stabilization at unobservable points: Analysis via an example. In 2017 IEEE 56th Annual Conference on Decision and Control (CDC), pages 6744-6749, Dec 2017.

[15] L. Marconi, L. Praly, and A. Isidori. Output stabilization via nonlinear Luenberger observers. SIAM J. Control Optim., 45(6):2277-2298, 2007.

[16] H. Shim and A. Teel. Asymptotic controllability and observability imply semiglobal practical asymptotic stabilizability by sampled-data output feedback. Automatica, 39(3):441 $-454,2003$. 
[17] A. Teel and L. Praly. Global stabilizability and observability imply semi-global stabilizability by output feedback. Systems Control Lett., 22(5):313-325, 1994.

[18] A. Teel and L. Praly. Tools for semiglobal stabilization by partial state and output feedback. SIAM J. Control Optim., 33(5):1443-1488, 1995.

[19] M. Tucsnak and G. Weiss. Observation and Control for Operator Semigroups. Birkhäuser Advanced Texts / Basler Lehrbücher. Birkhäuser Verlag, 2009. 